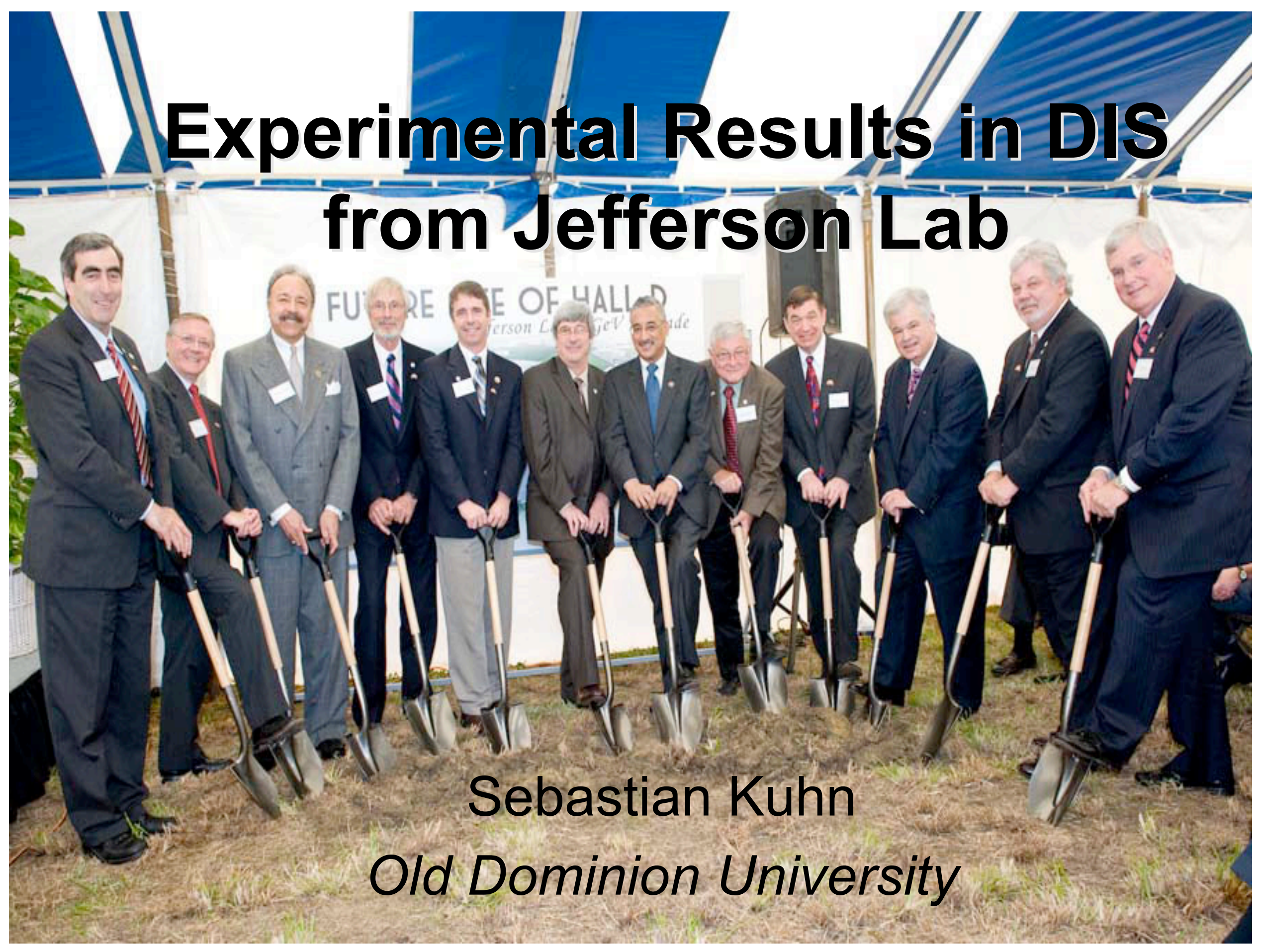




\section{Jefferson Lab in Perspective}

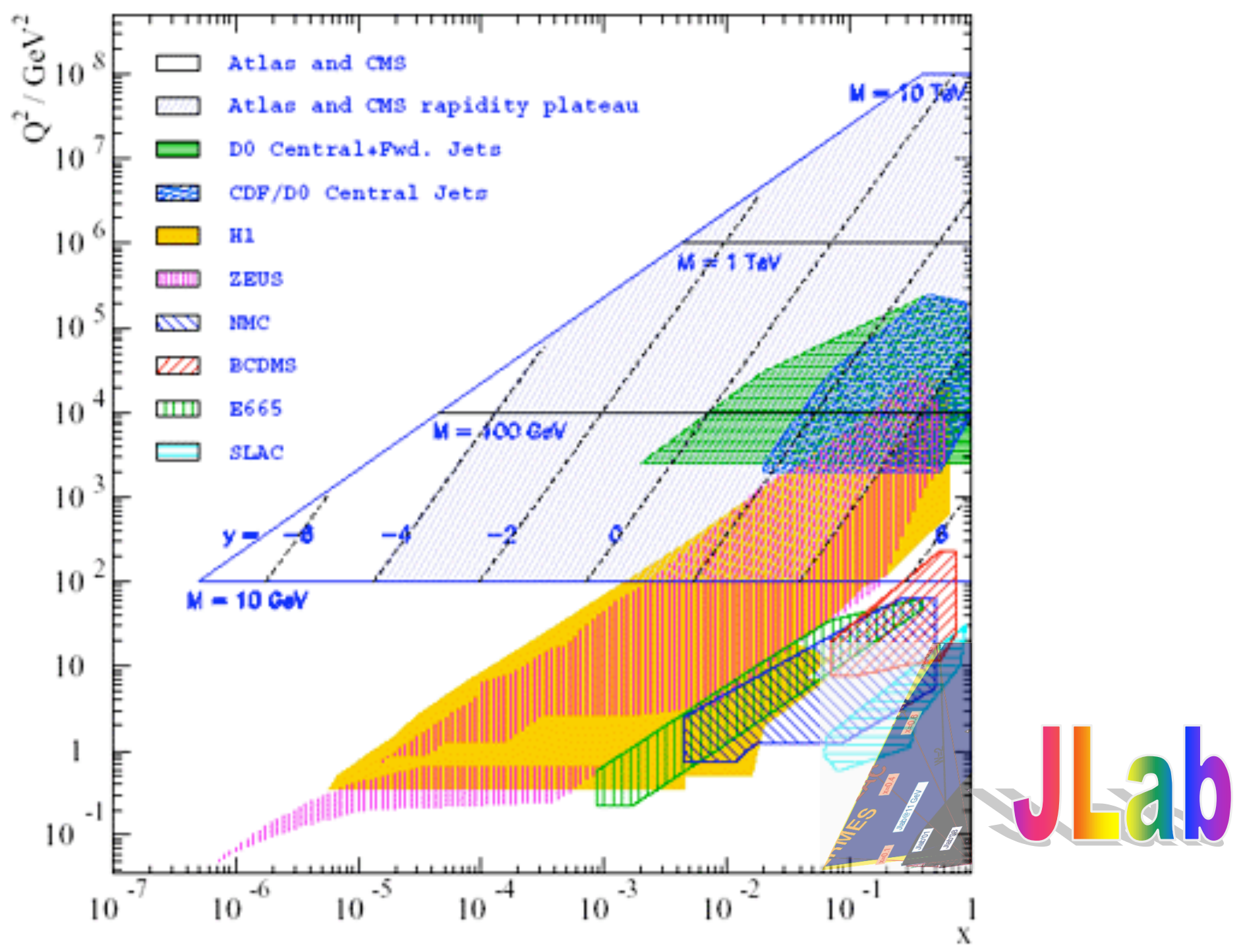




\section{Slightly Enlarged...}

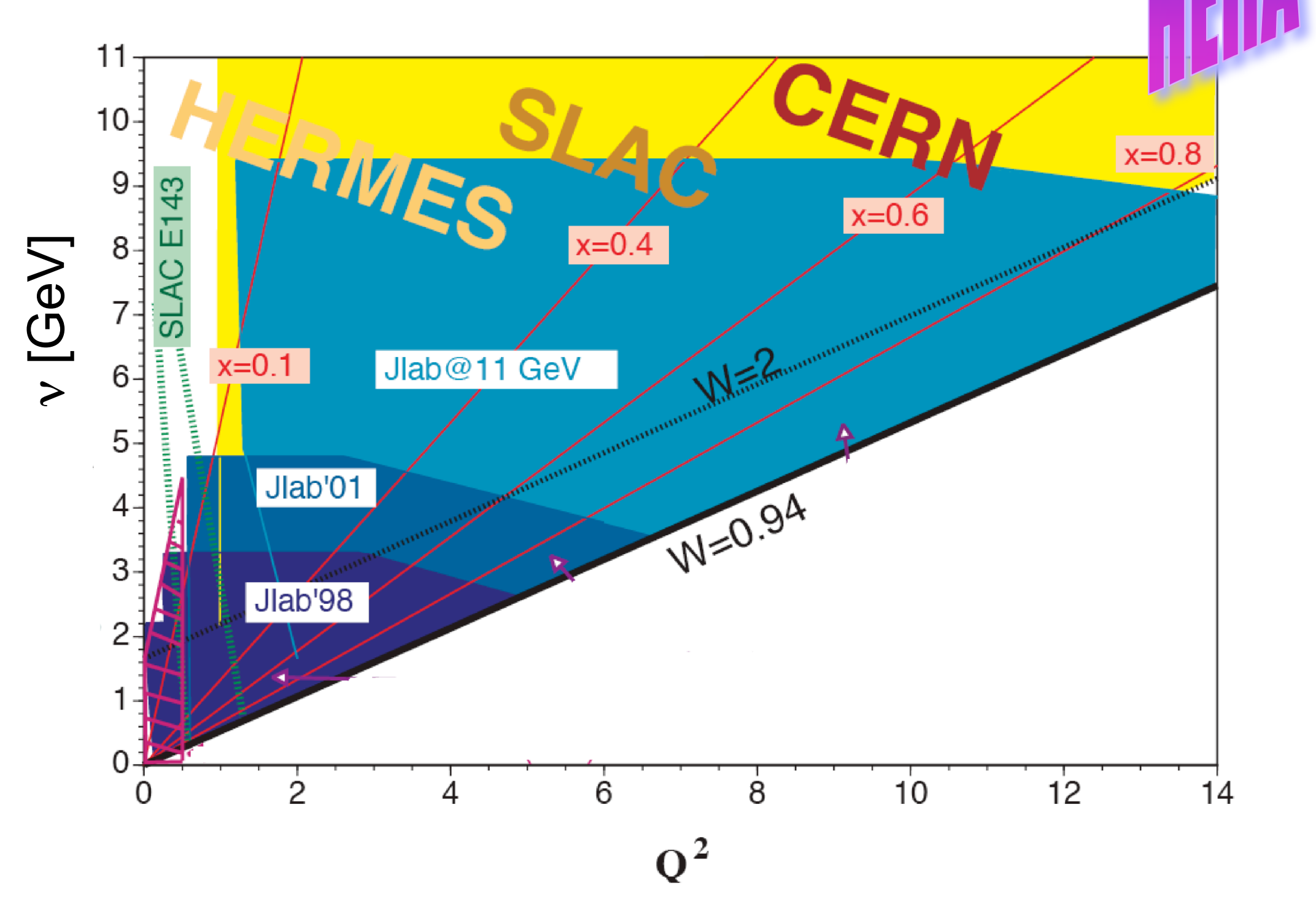




\section{Topics JLab Can Address:}

- Inclusive (D)IS

- Valence PDFs (d/u, $\Delta \mathrm{d} / \mathrm{d}$ and $\Delta \mathrm{u} / \mathrm{u}$ as $\mathrm{x} \rightarrow 1$ )

- QCD evolution, higher twist, duality

- Moments, sum rules, OPE

- Structure of bound nucleons

- Semi-inclusive DIS

- Tagged (spin) structure functions

- Transversity, TMD PDFs (Single Spin Asymmetries)

- Hard Exclusive Processes

- Generalized PDs

- DVCS, Deeply virtual meson production 


\section{Experimental Hall A}
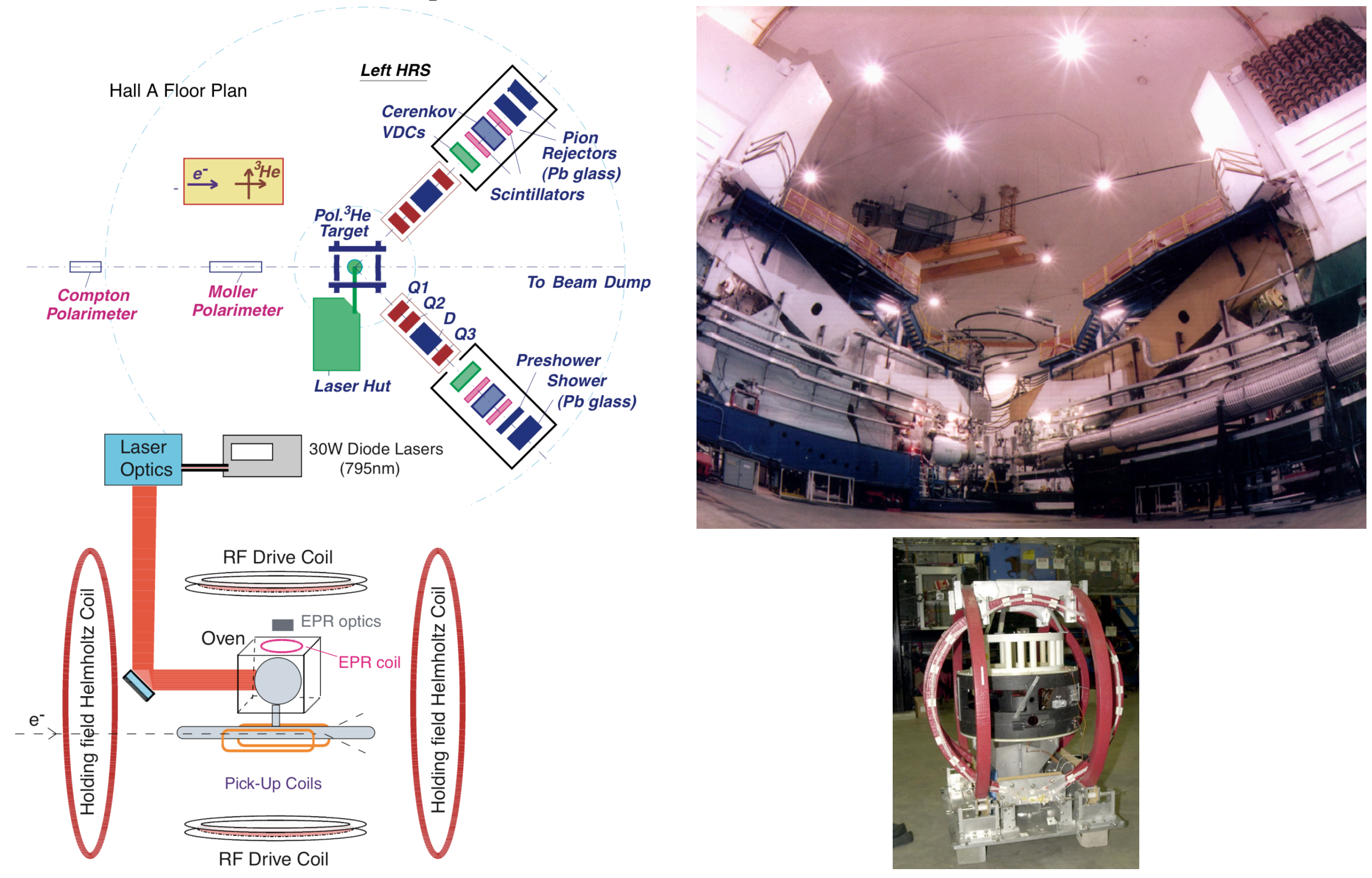


\section{Experimental Hall B}
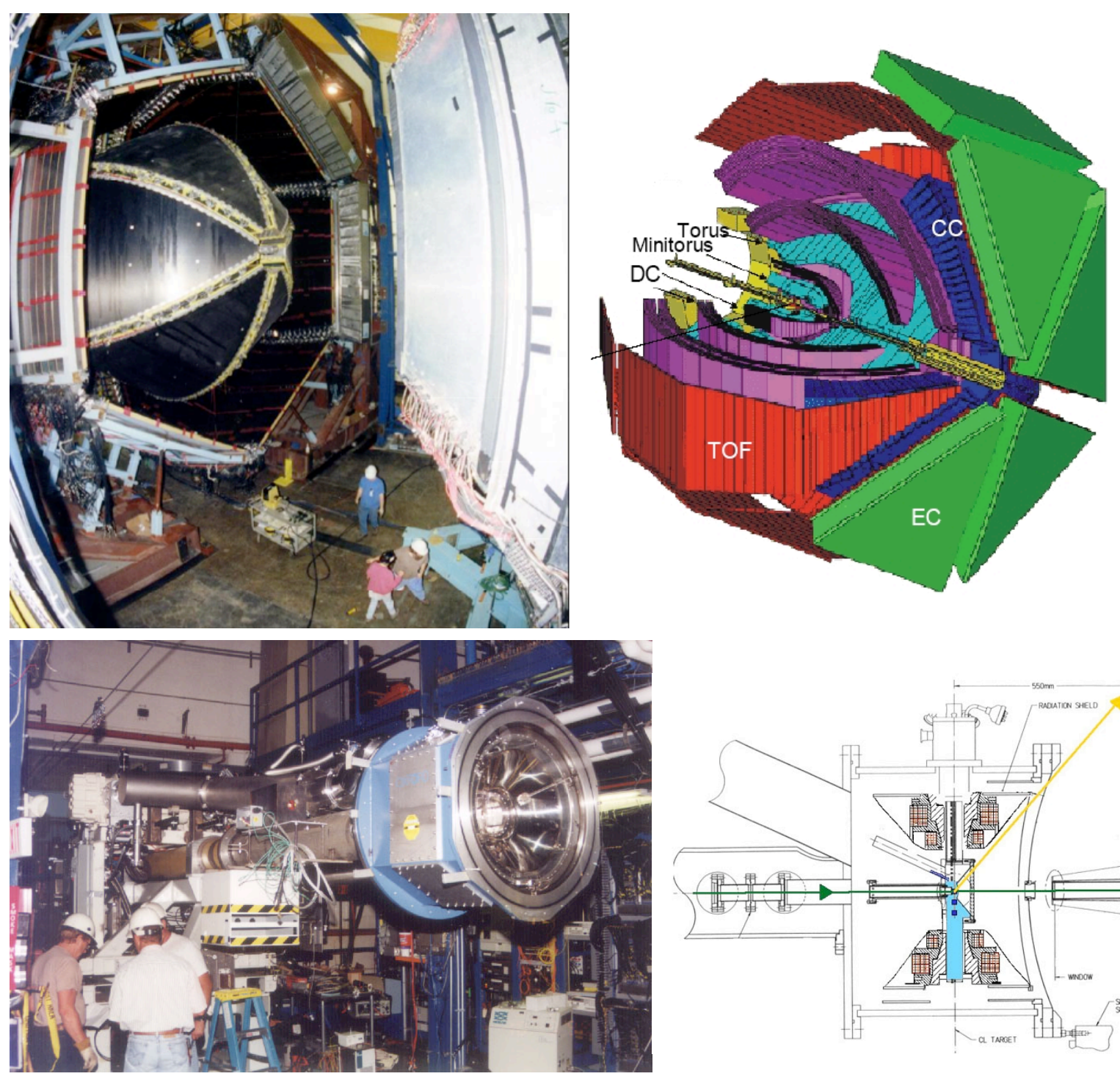

\section{CLAS}

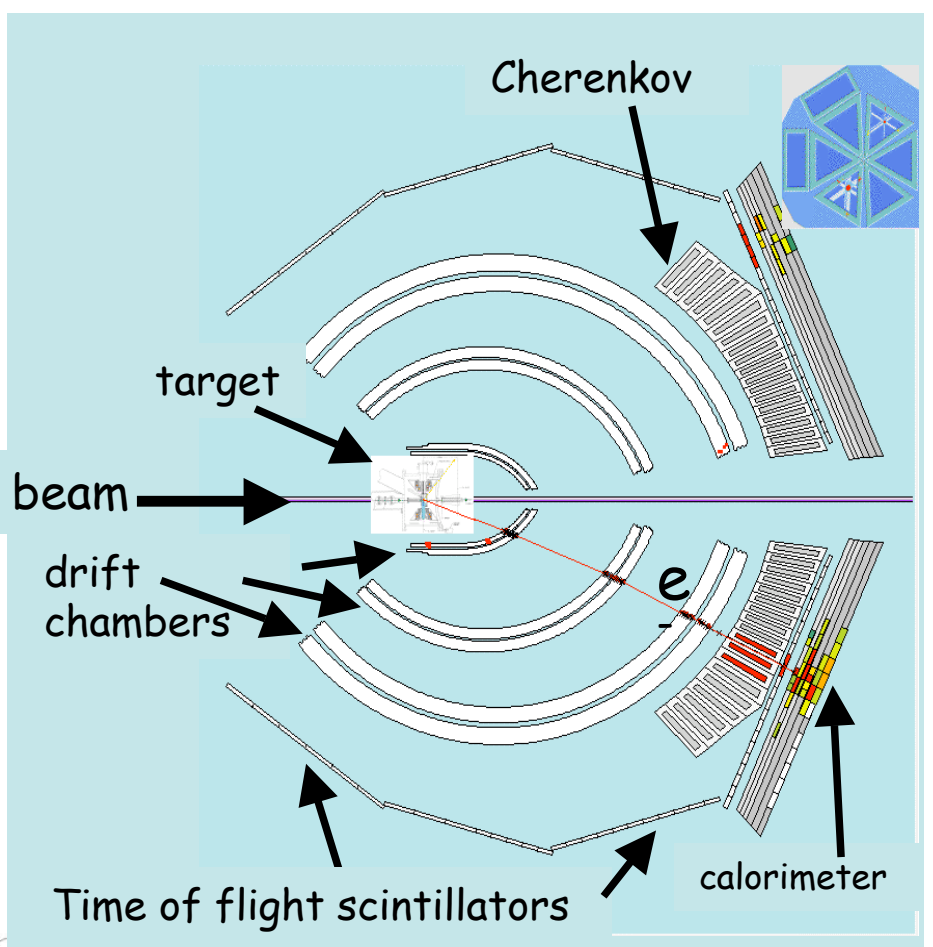




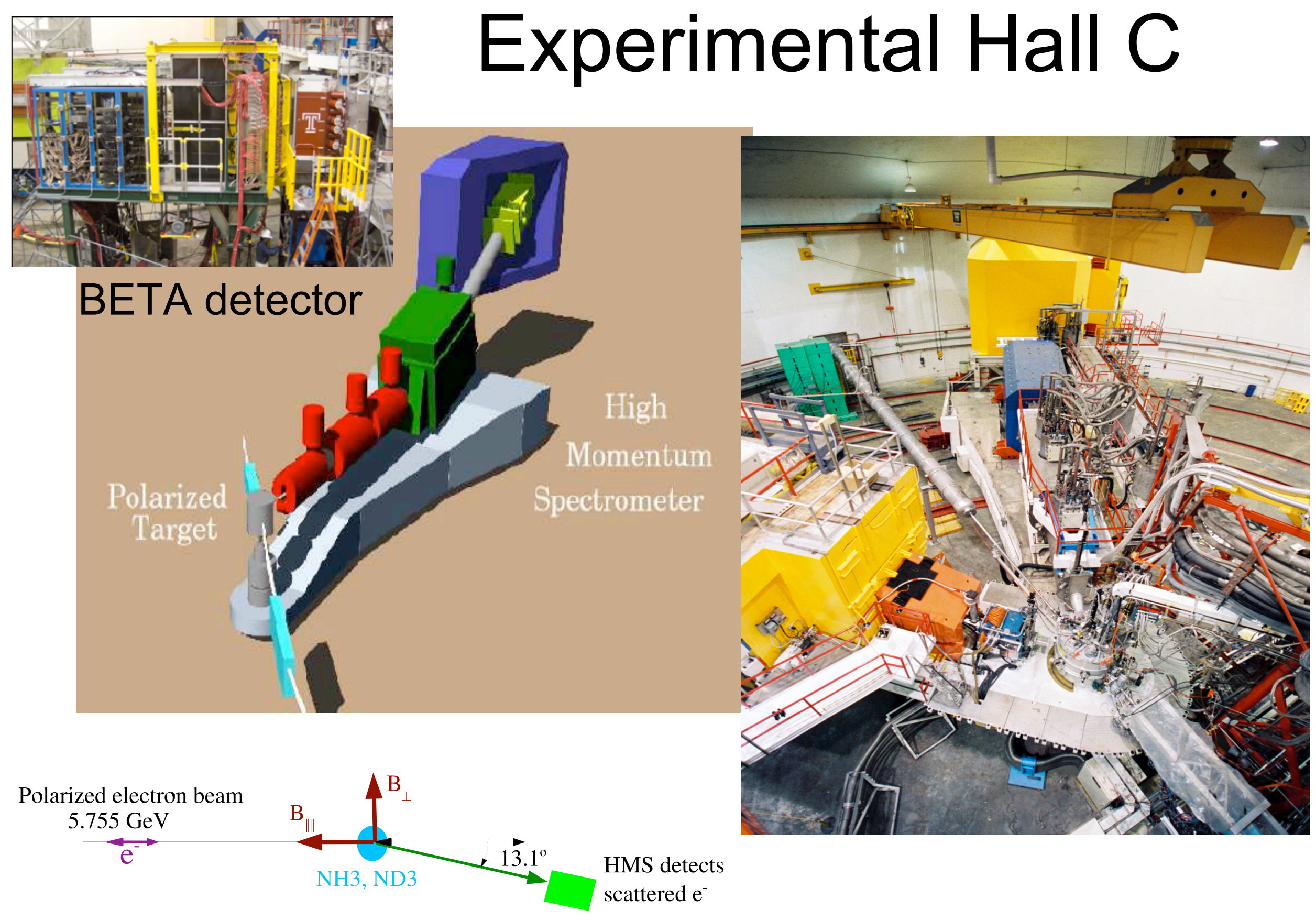




\section{Structure Functions - Bread \& Butter (Wine?)}

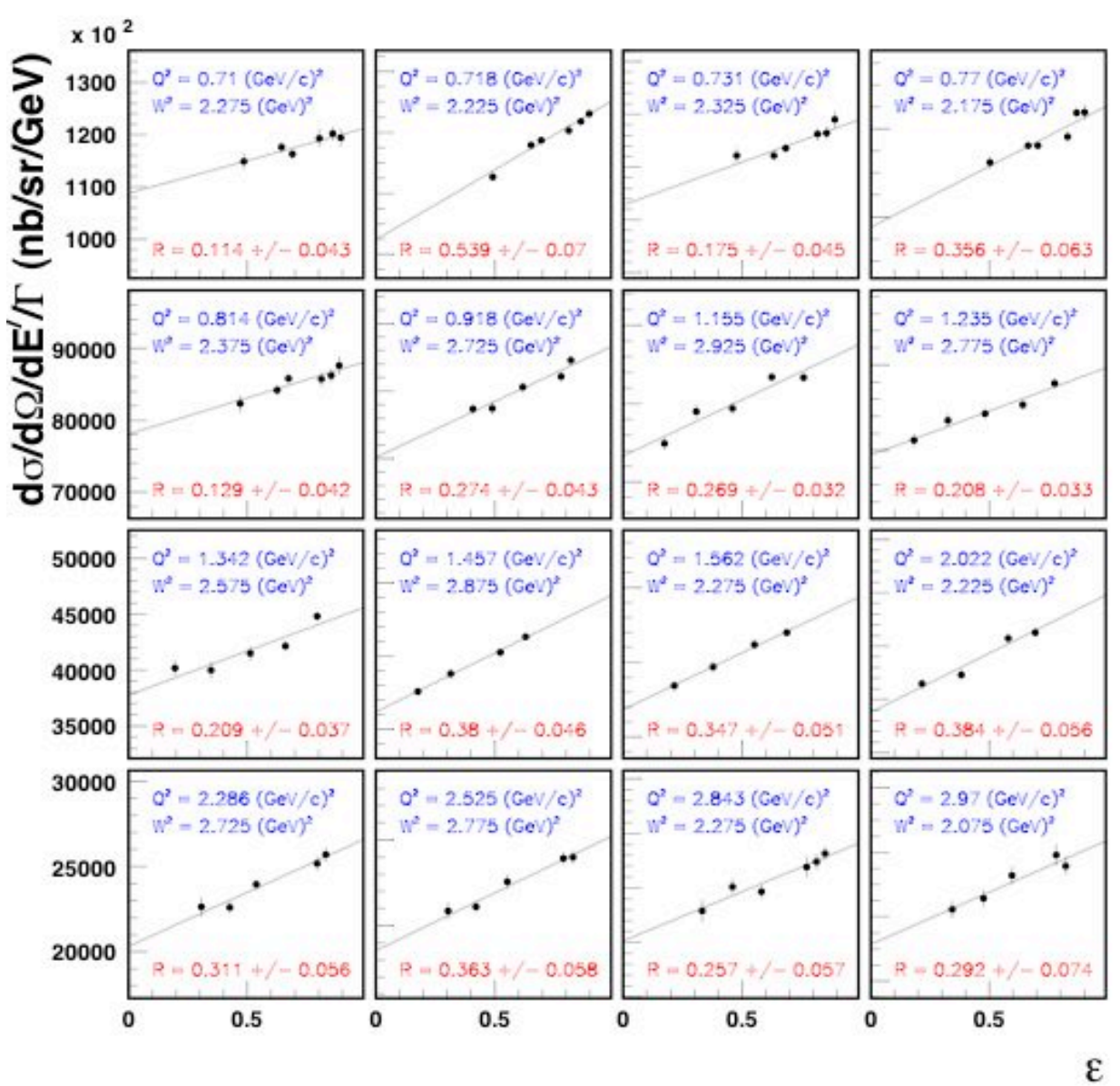

High Precision L/T separation in Hall C

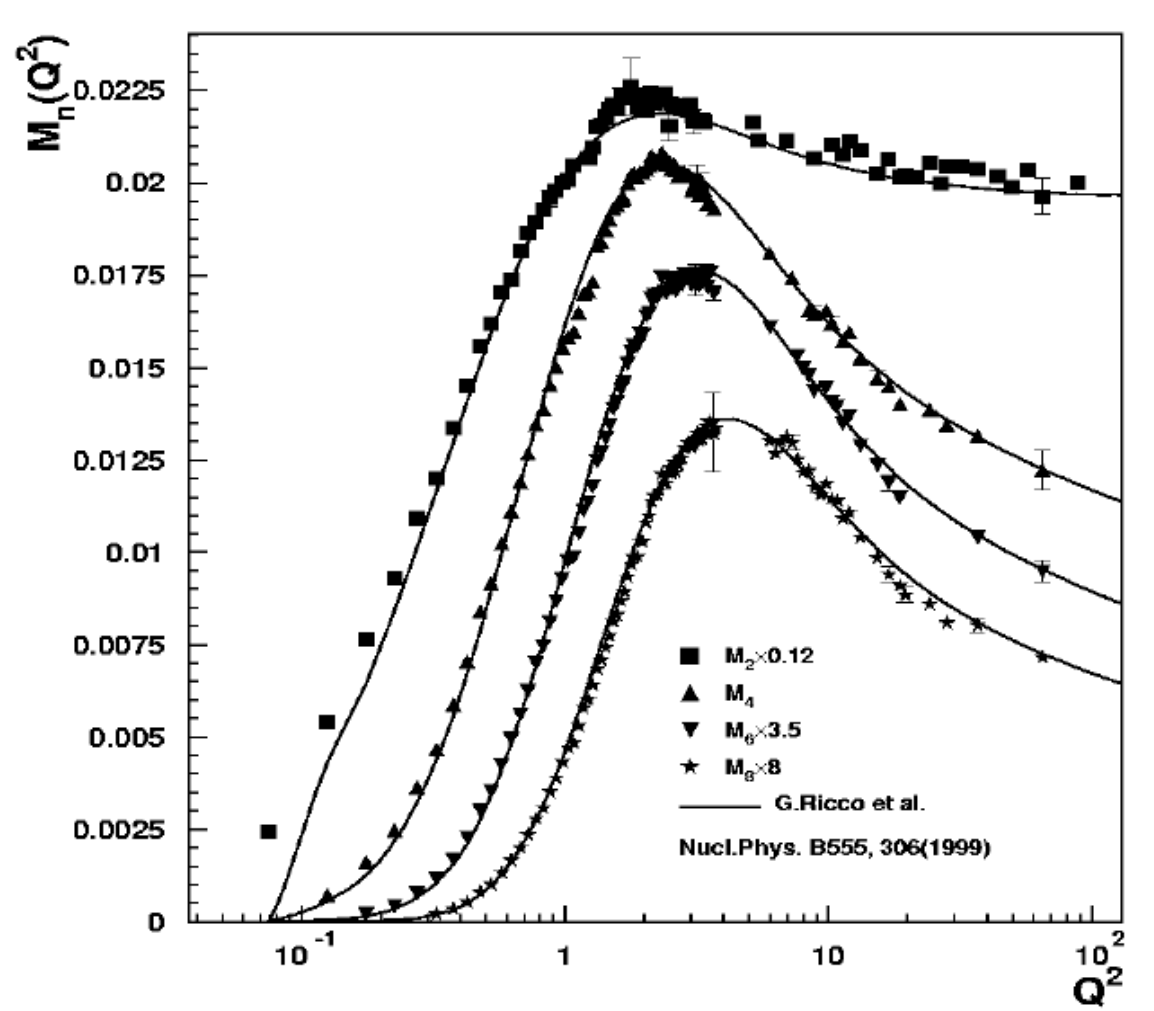

Nachtmann Moments Global analysis by M. Osipenko (including CLAS data) 


\section{Valence PDFs}

- Behavior of PDFs still unknown for $x \rightarrow 1$

- $S U(6): d / u=1 / 2, \Delta u / u=2 / 3, \Delta d / d=-1 / 3$ for all $x$

- Relativistic Quark model: $\Delta \mathrm{u}, \Delta \mathrm{d}$ reduced

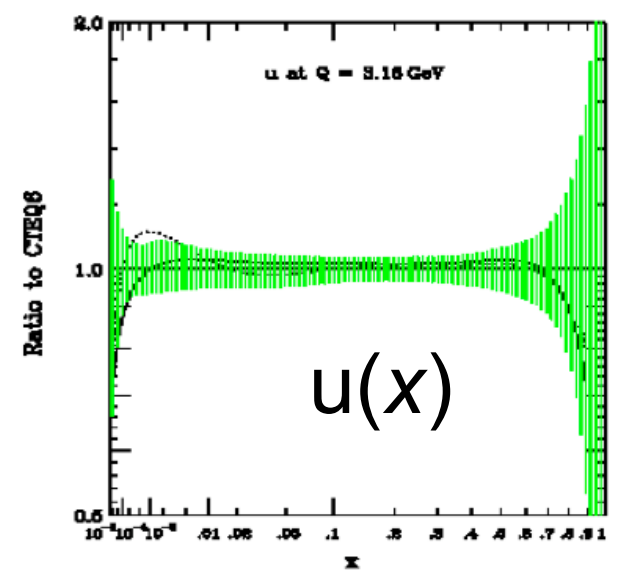

- Hyperfine effect (1-gluon-exchange): Spectator spin 1 suppressed, $\mathrm{d} / \mathrm{u}=0, \Delta \mathrm{u} / \mathrm{u}=1, \Delta \mathrm{d} / \mathrm{d}=-1 / 3$

- Helicity conservation: $\mathrm{d} / \mathrm{u}=1 / 5, \Delta \mathrm{u} / \mathrm{u}=1, \Delta \mathrm{d} / \mathrm{d}=1$

- Orbital angular momentum: can explain slower convergence to $\Delta \mathrm{d} / \mathrm{d}=1$

- $d / u$ knowledge limited by lack of free neutron target (nuclear binding effects in $\mathrm{d},{ }^{3} \mathrm{He}$ )

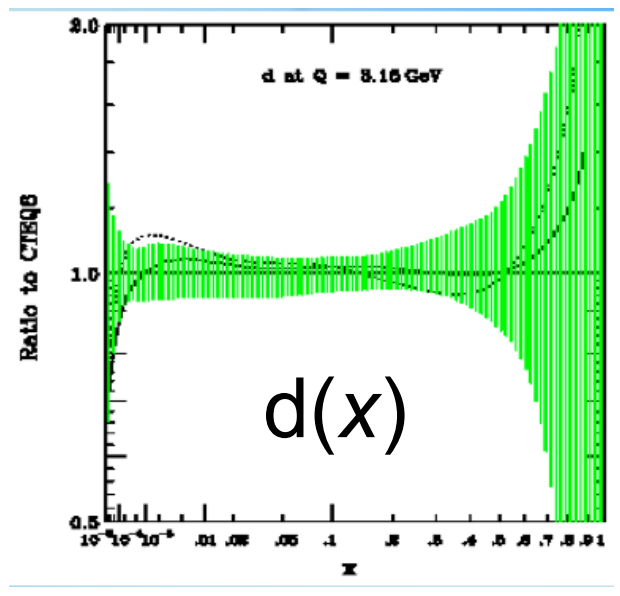

- Large $x$ requires very high luminosity

$\rightarrow$ See also C. Keppel's talk Session SF-9 


\section{d/u: The BoNuS Approach}

\section{"Barely Off-shell NeUtron Scattering"}

The Problem: nuclear binding uncertainties prevent us from knowing $\mathrm{F}_{2 \mathrm{n}}$ and $\mathrm{d} / \mathrm{u}(\mathrm{x} \rightarrow 1)$

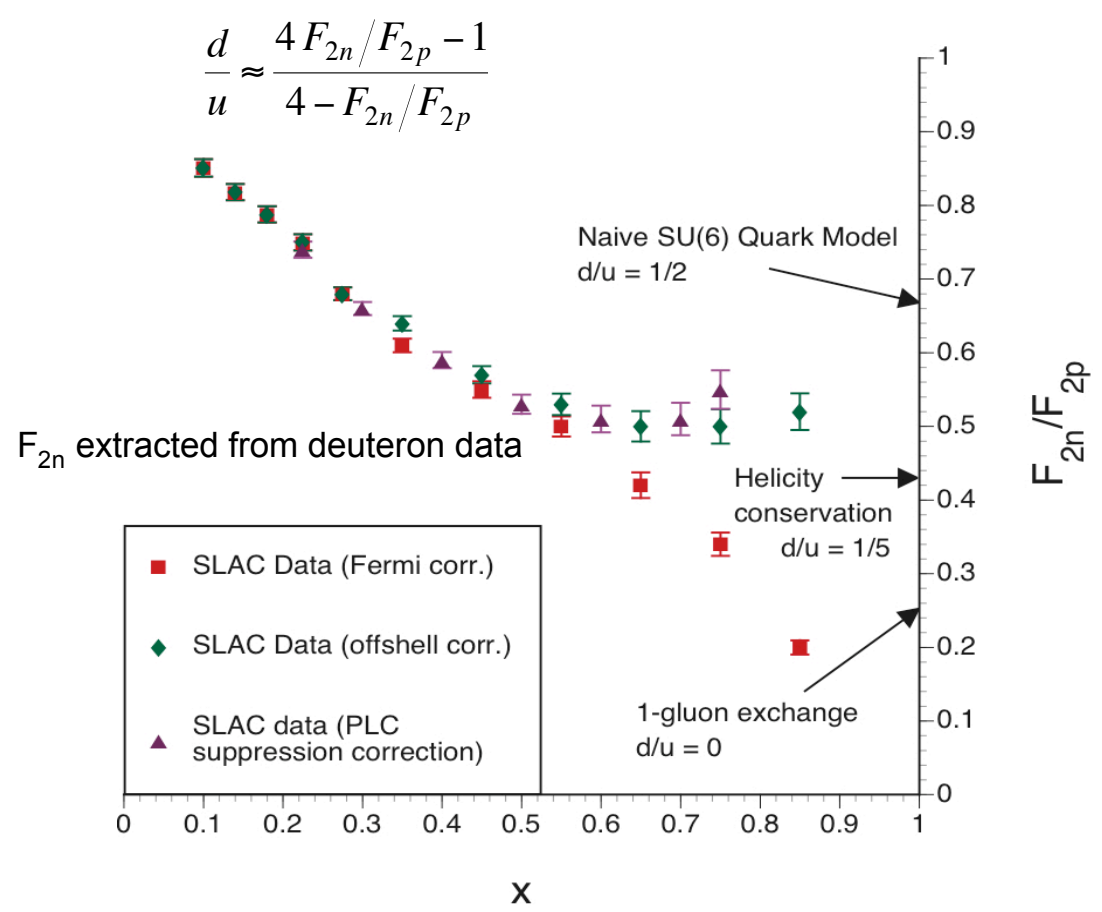

Require low $p_{\mathrm{s}}$ to suppress $\mathrm{FSI}$, binding and off-shell effects
The Solution: spectator proton tagging $\rightarrow$ select low-momentum part of $d$ wave function and tag motion of struck neutron

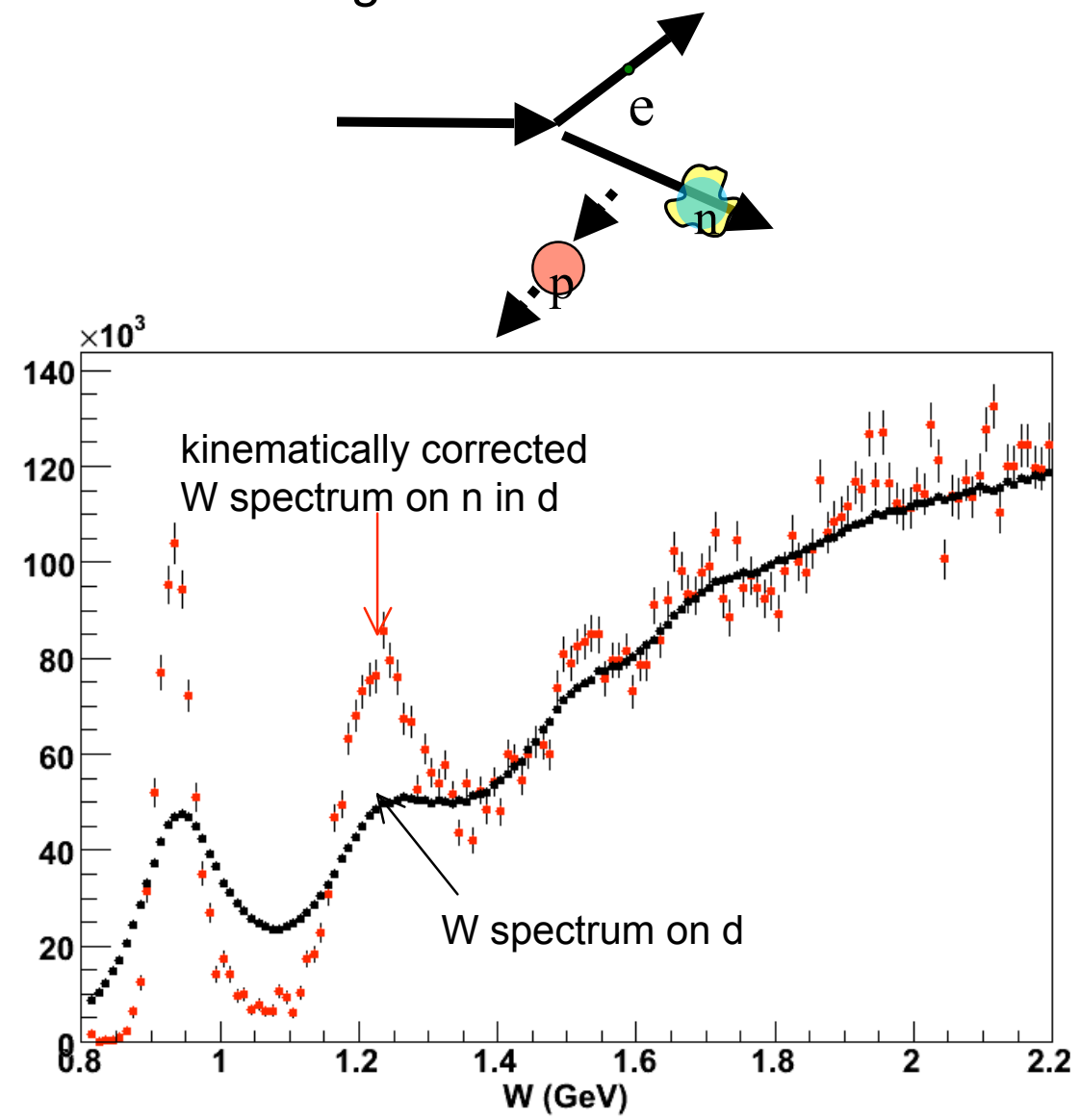




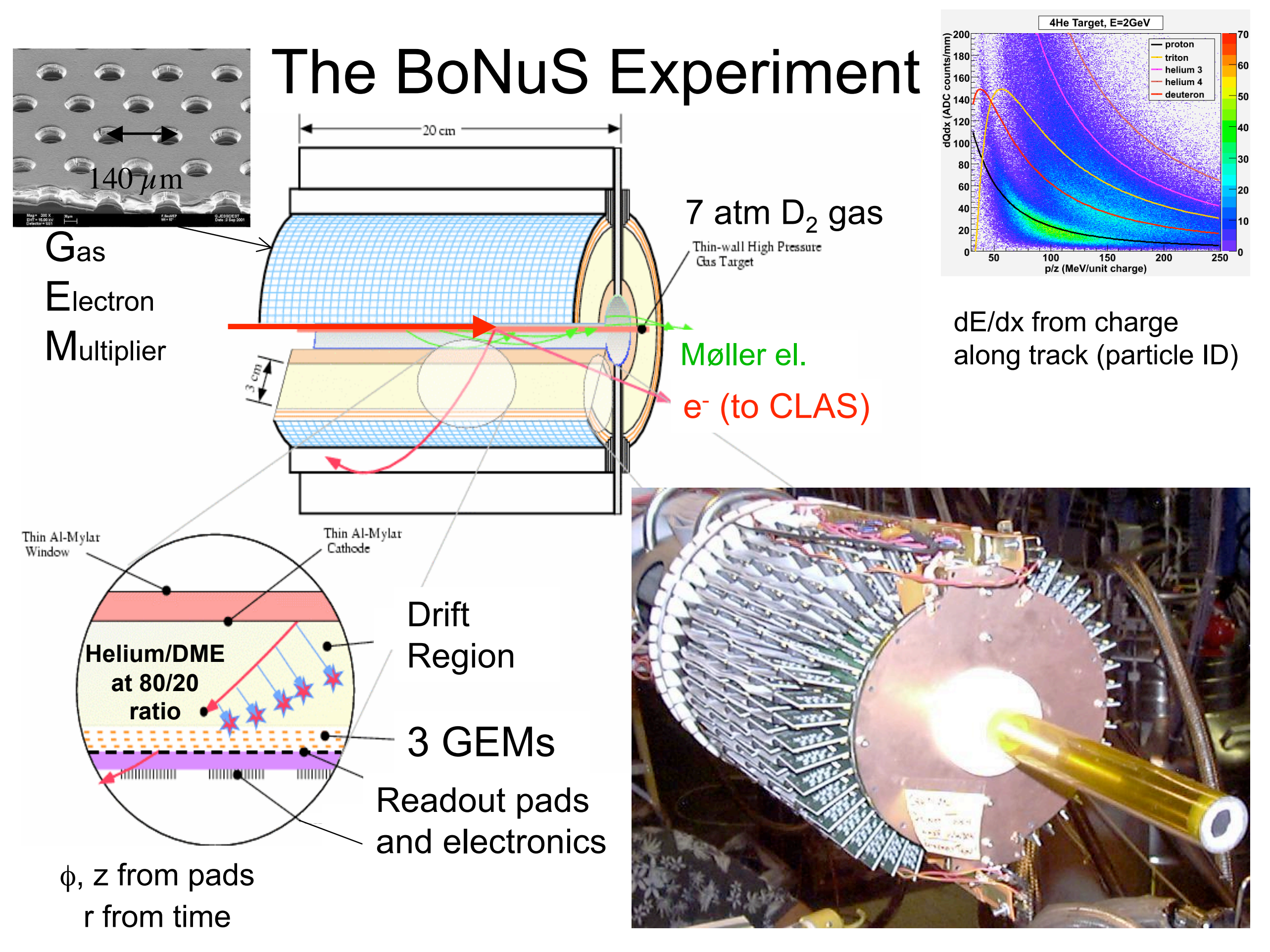




\section{BoNuS - Preliminary Results}

and Future
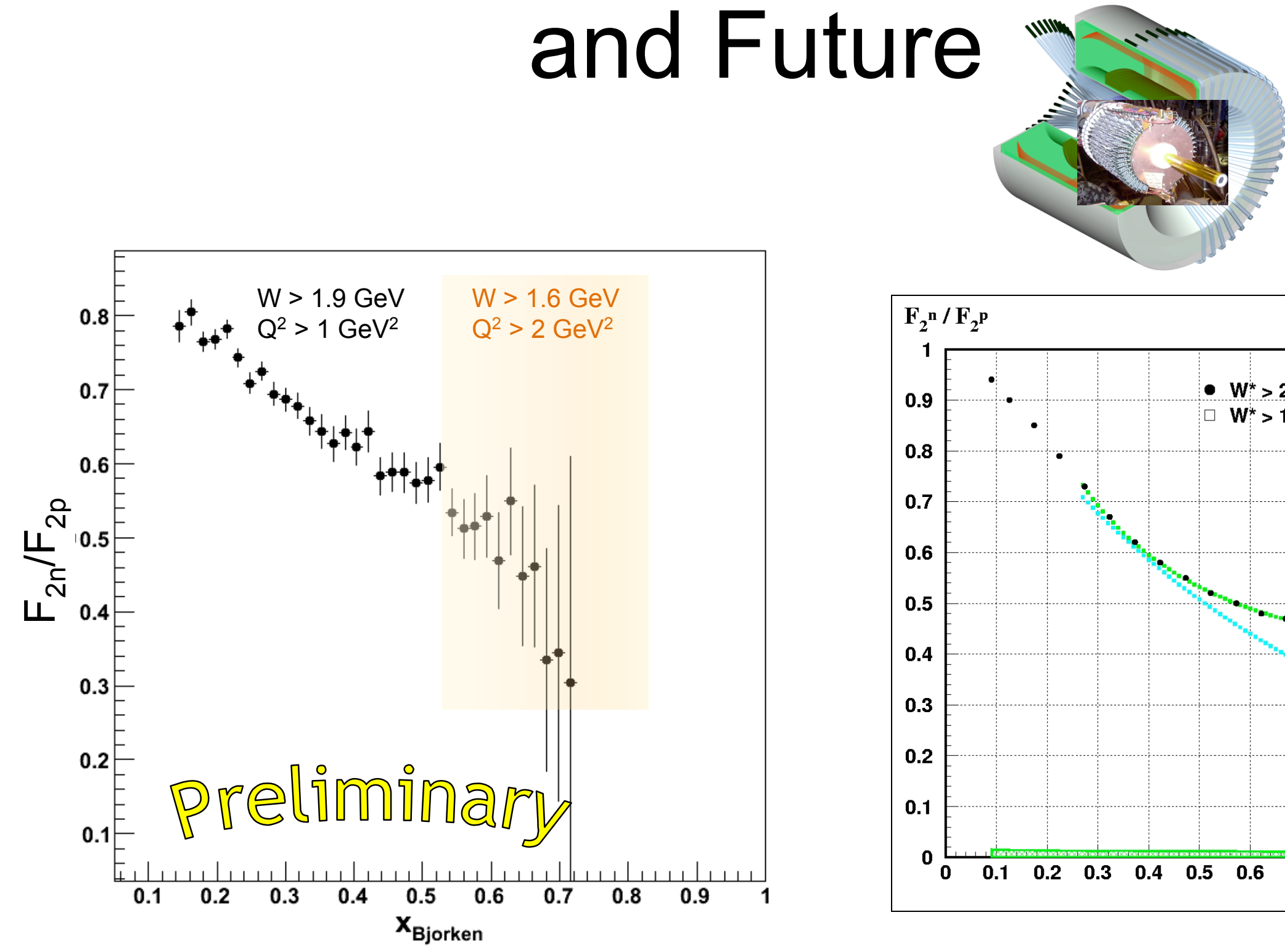

CLAS12

Central

Detector

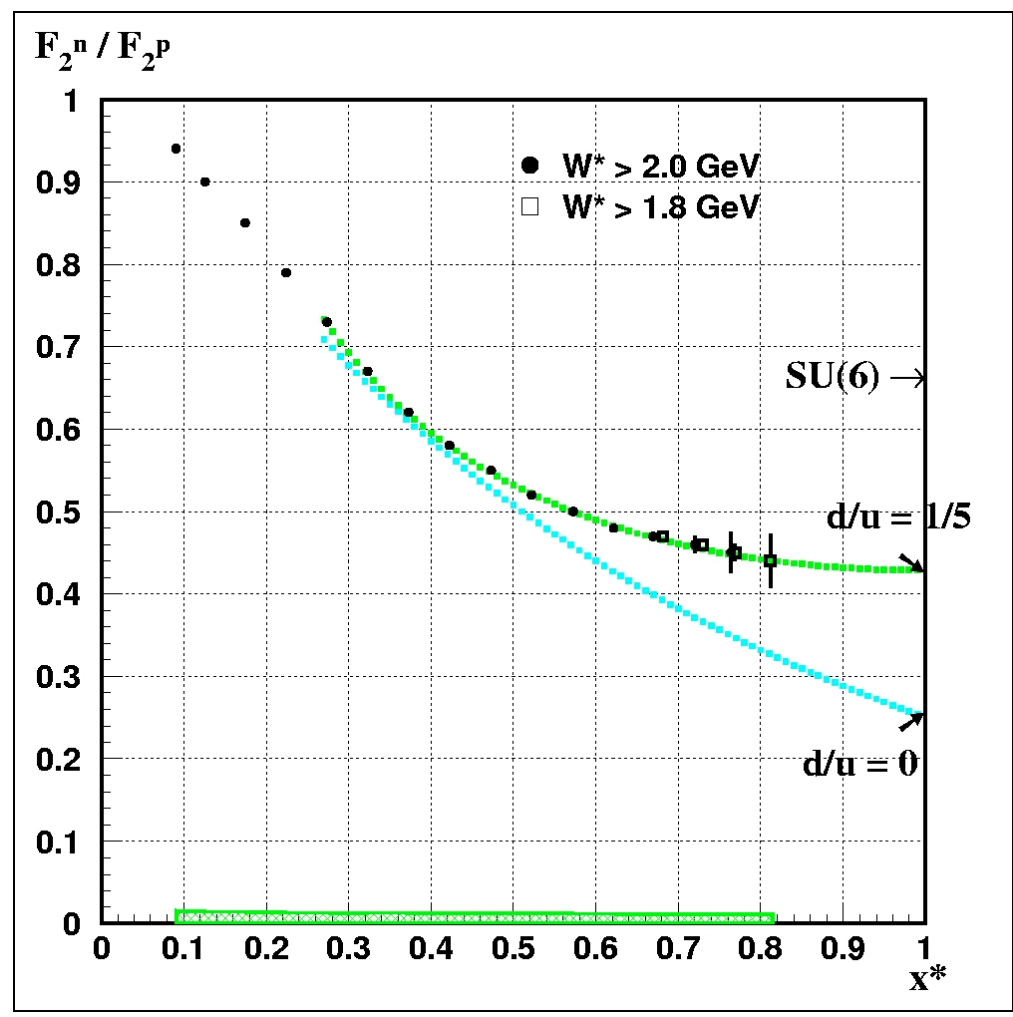




\section{Spin Structure Functions at large $x$}

Hall A
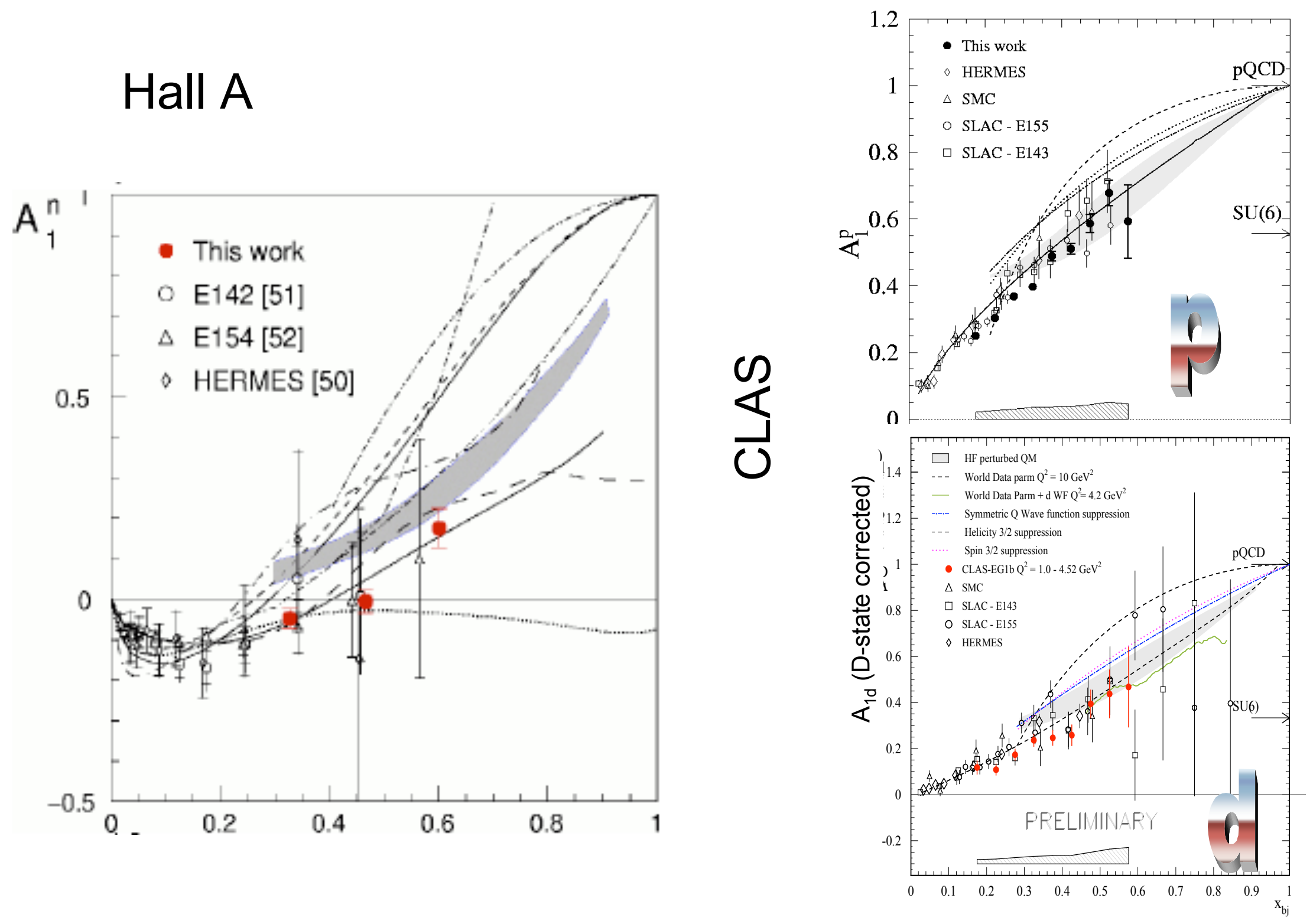


\section{Results and Future}

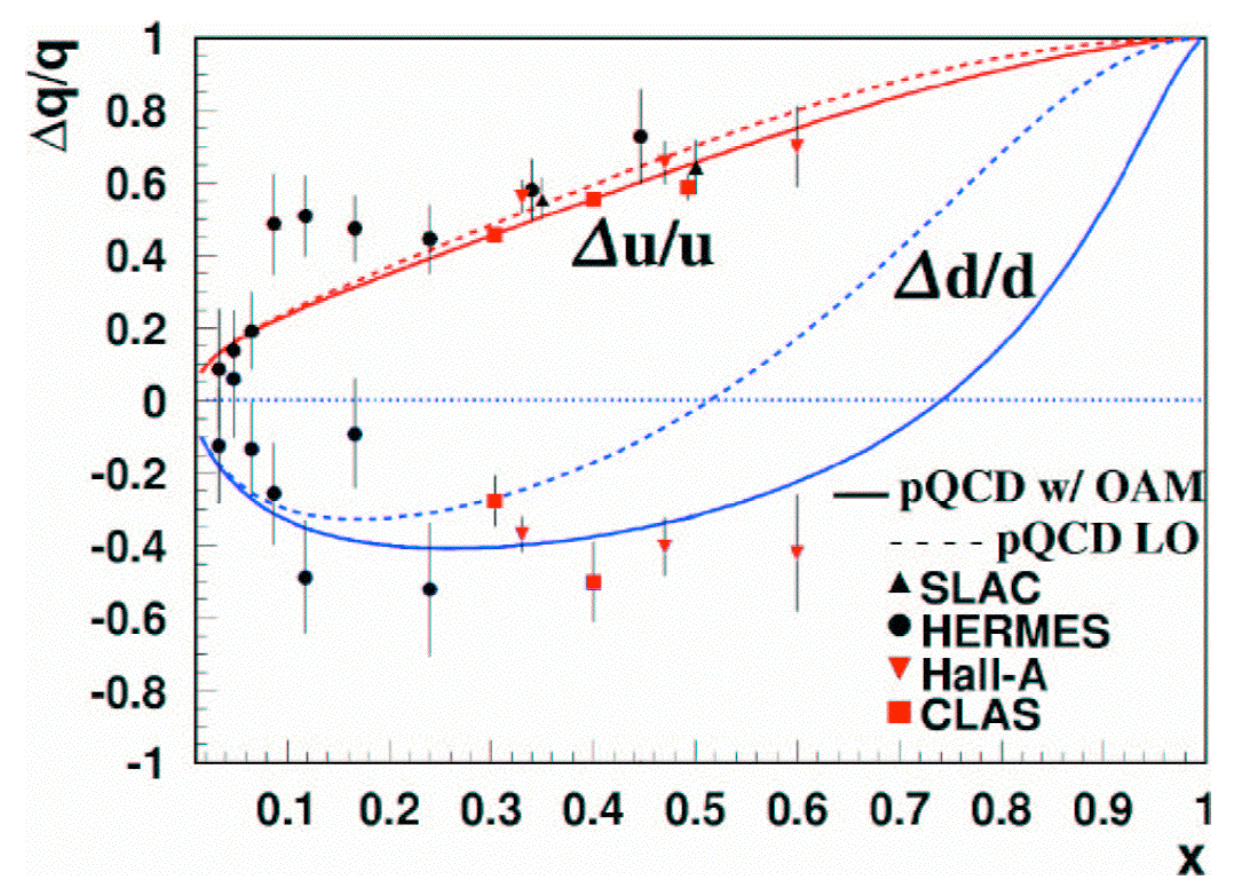

Avakian et al., Phys.Rev.Lett.99:082001,2007
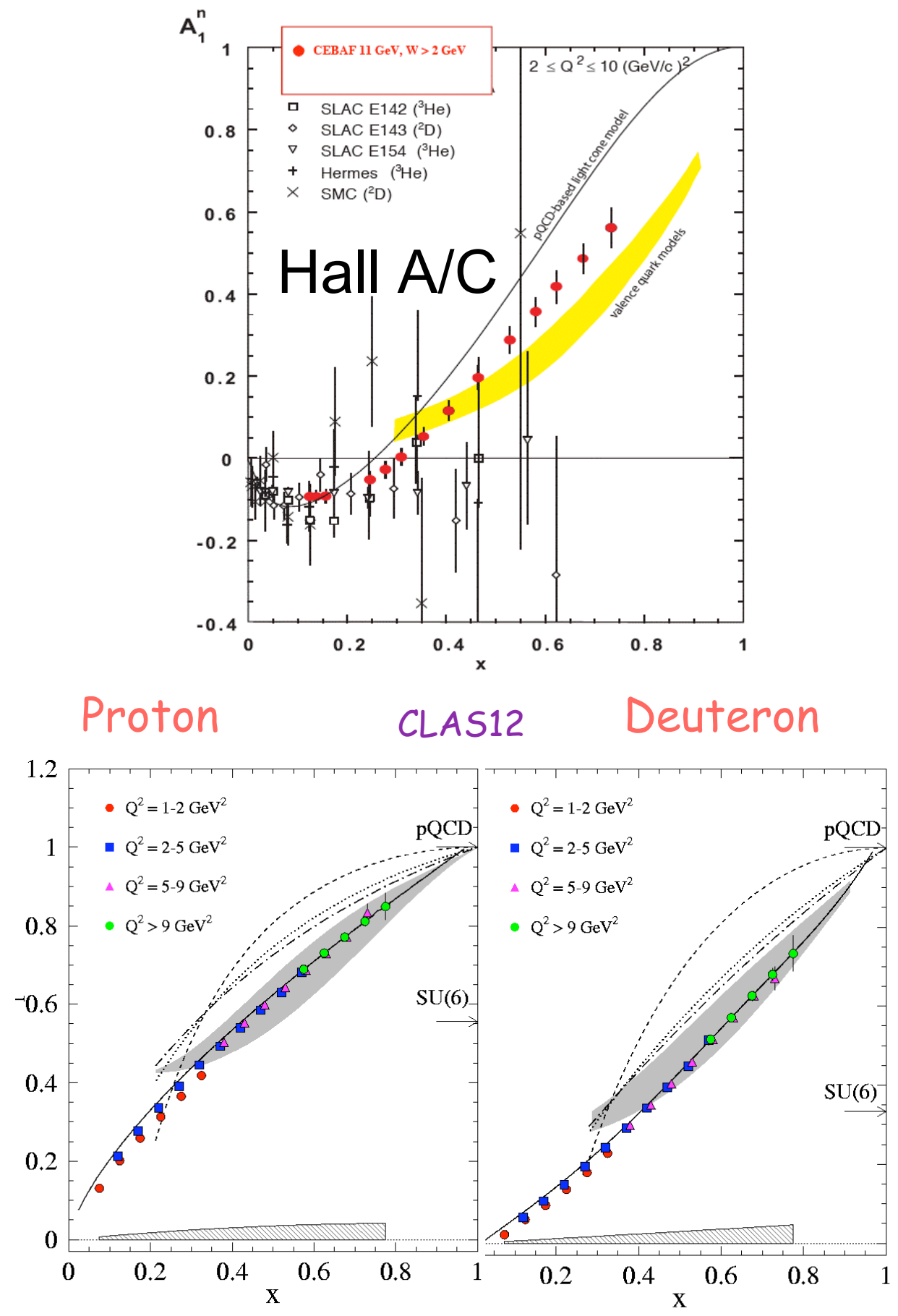


\section{Huge number of data points on $g_{1}, g_{2}, \ldots$}

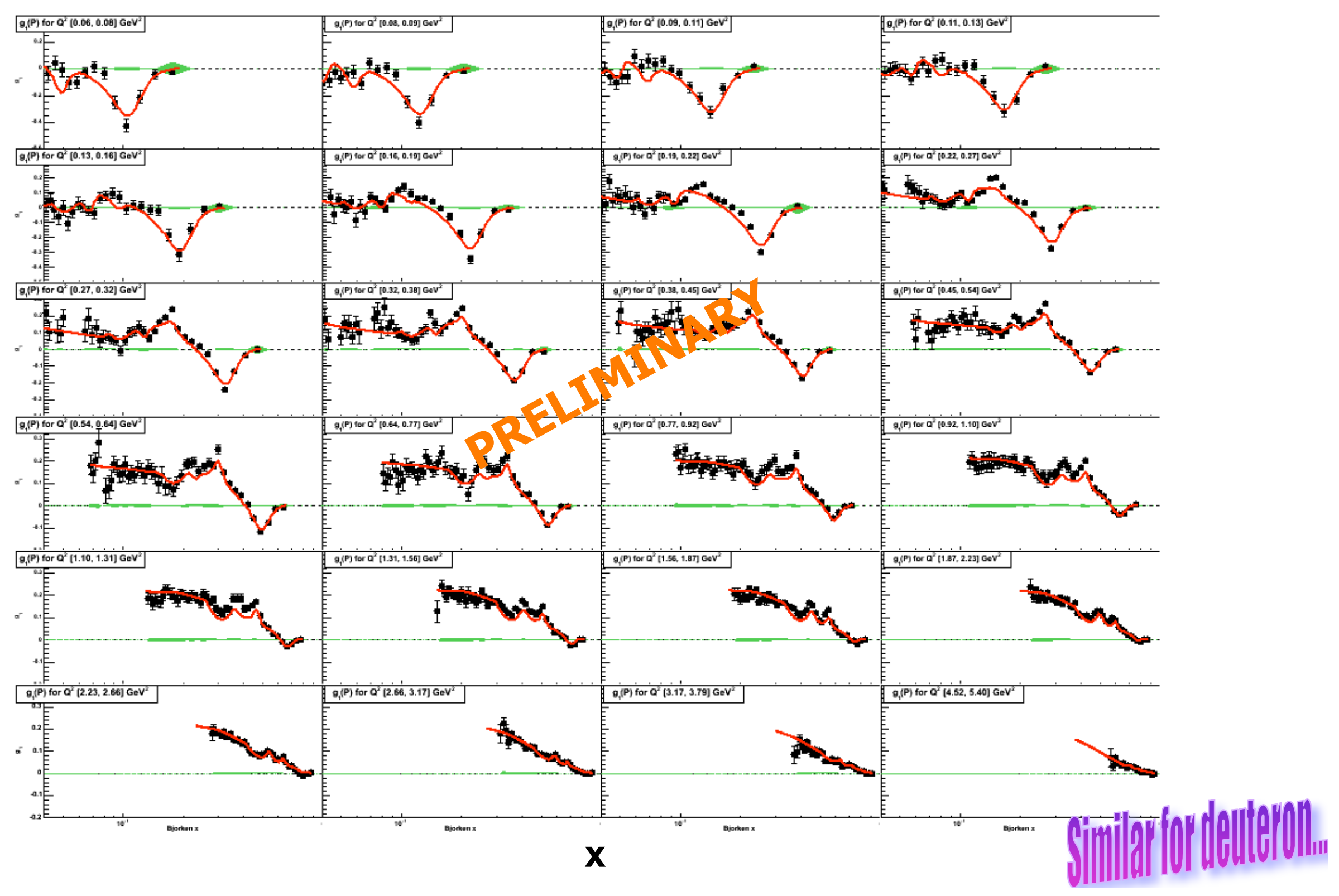




\section{...with impact on PDFs}

$g_{1}\left(x, Q^{2}\right)_{p Q C D}=\frac{1}{2} \sum_{q}^{N_{f}} e_{q}^{2}\left[(\Delta q+\Delta \bar{q}) \otimes\left(1+\frac{\alpha_{s}\left(Q^{2}\right)}{2 \pi} \delta C_{q}\right)+\frac{\alpha_{s}\left(Q^{2}\right)}{2 \pi} \Delta G \otimes \frac{\delta C_{G}}{N_{f}}\right]$
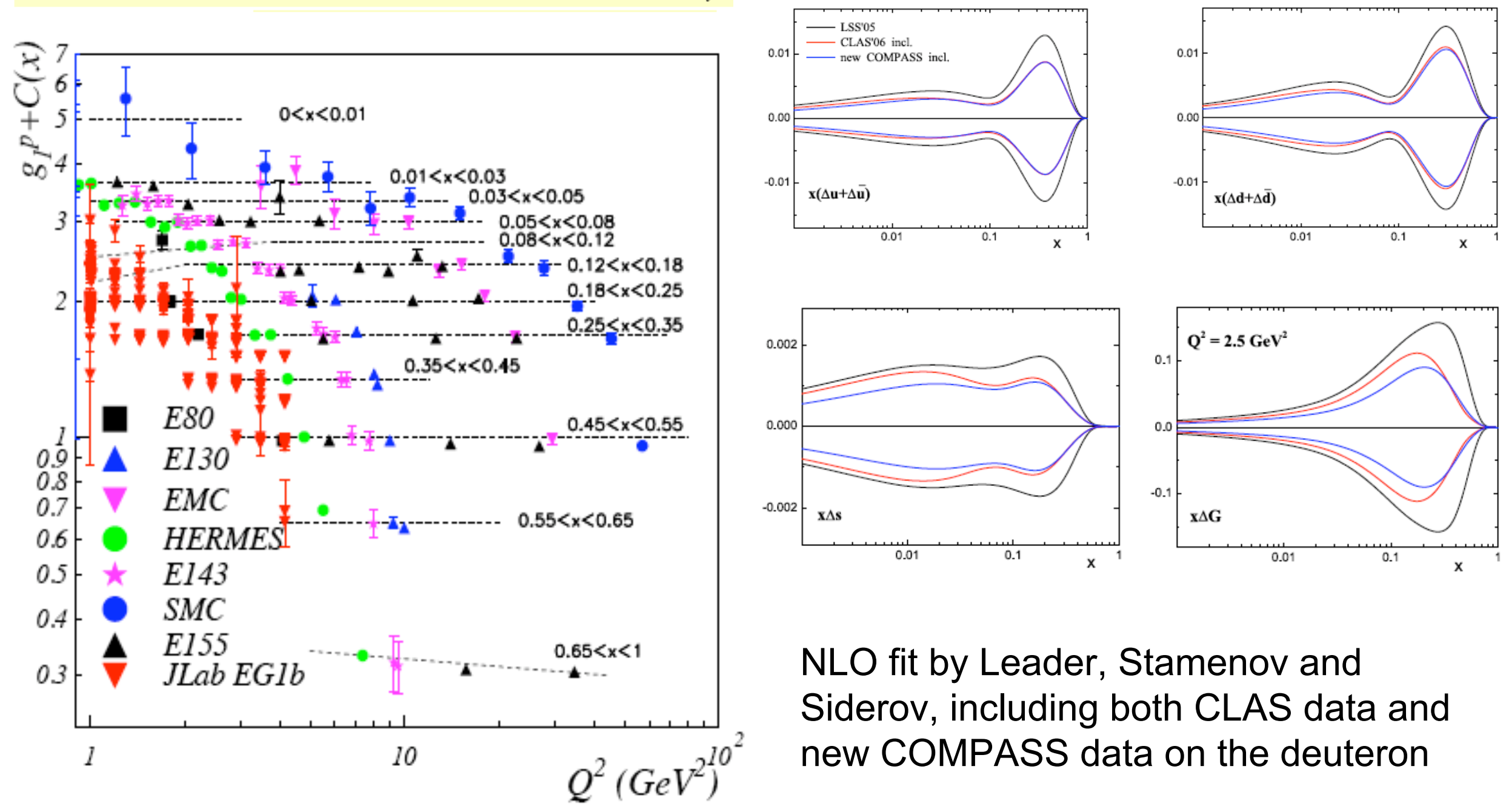

NLO fit by Leader, Stamenov and Siderov, including both CLAS data and new COMPASS data on the deuteron 


\section{The Spin Structure Function $\mathrm{g}_{2}$}
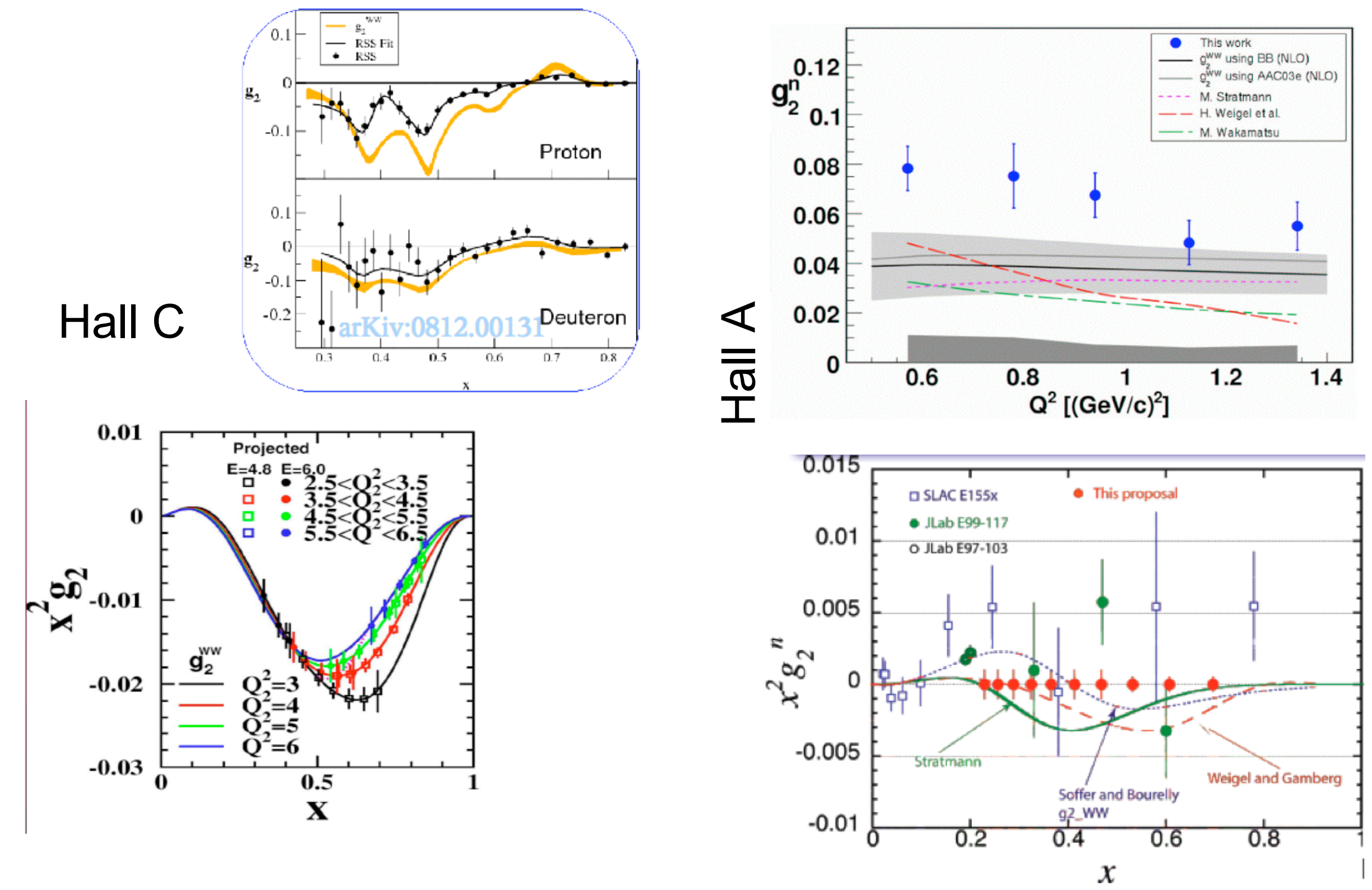


\section{Moments: Spin Structure Functions}
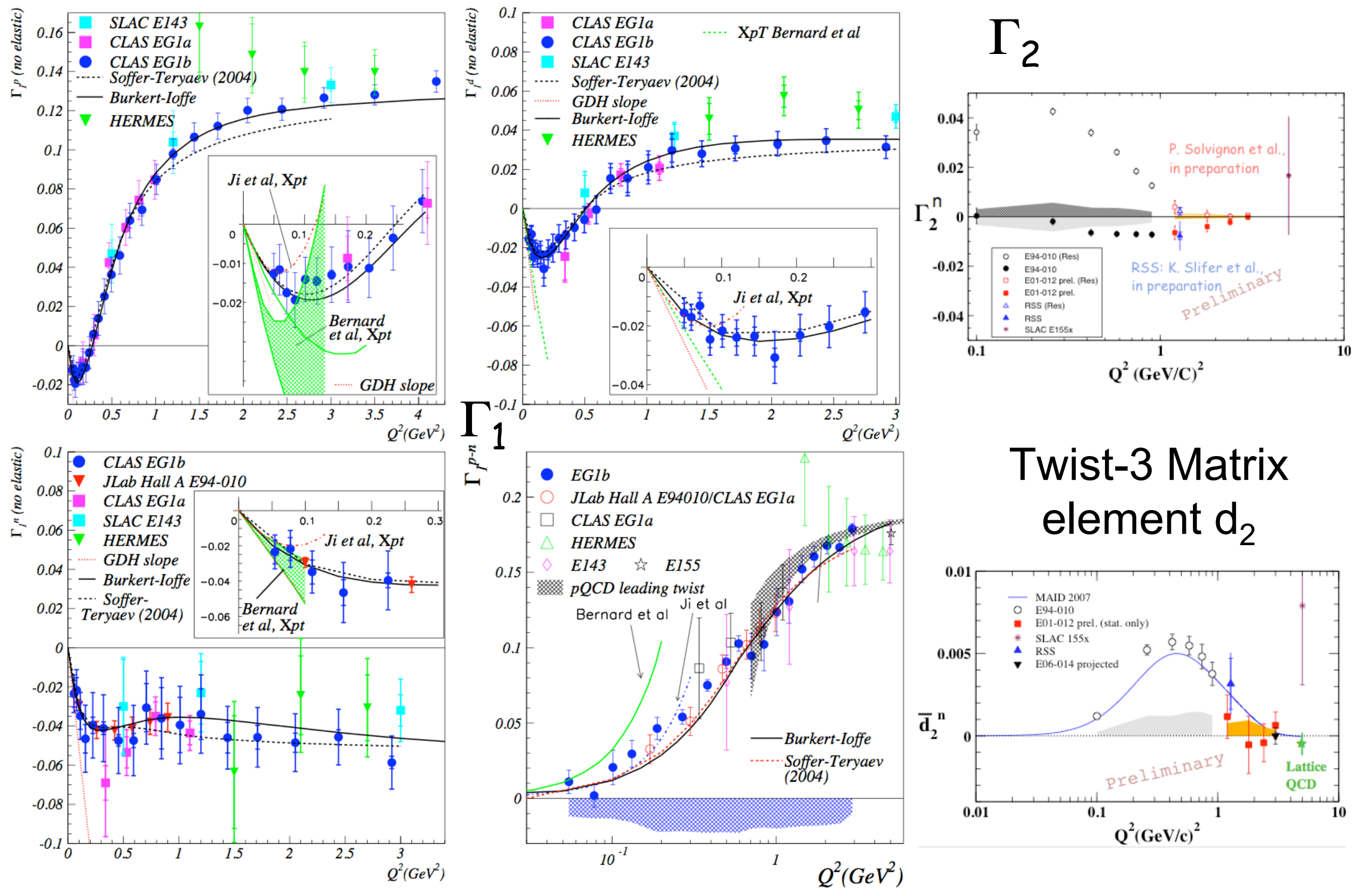

\section{Twist-3 Matrix element $d_{2}$}

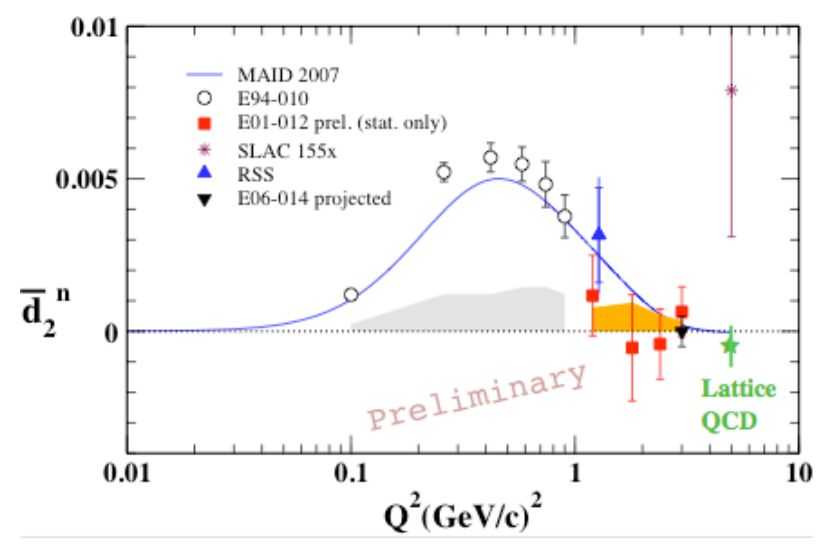




\section{Higher Twist}

$\Gamma_{1}\left(Q^{2}\right)=\mu_{2}\left(\ln Q^{2}\right)+\frac{\mu_{4}\left(\ln Q^{2}\right)}{Q^{2}}+\cdots ; \quad \mu_{4}=\frac{M^{2}}{9}\left(a_{2}+4 d_{2}+4 f_{2}\right)$

twist-2, targ. mass

$d_{2}\left(Q^{2}\right)=\int_{0}^{1} x^{2}\left[2 g_{1}\left(x, Q^{2}\right)+3 g_{2}\left(x, Q^{2}\right)\right] d x \quad$ twist-3

$f_{2}\left(Q^{2}\right) M^{2} S^{\mu}=\frac{1}{2} \sum_{q} e_{q}^{2}\left\langle N\left|g \bar{\psi}_{q} \widetilde{G}^{\mu \nu} \gamma_{\nu} \psi_{q}\right| N\right\rangle$ twist-4
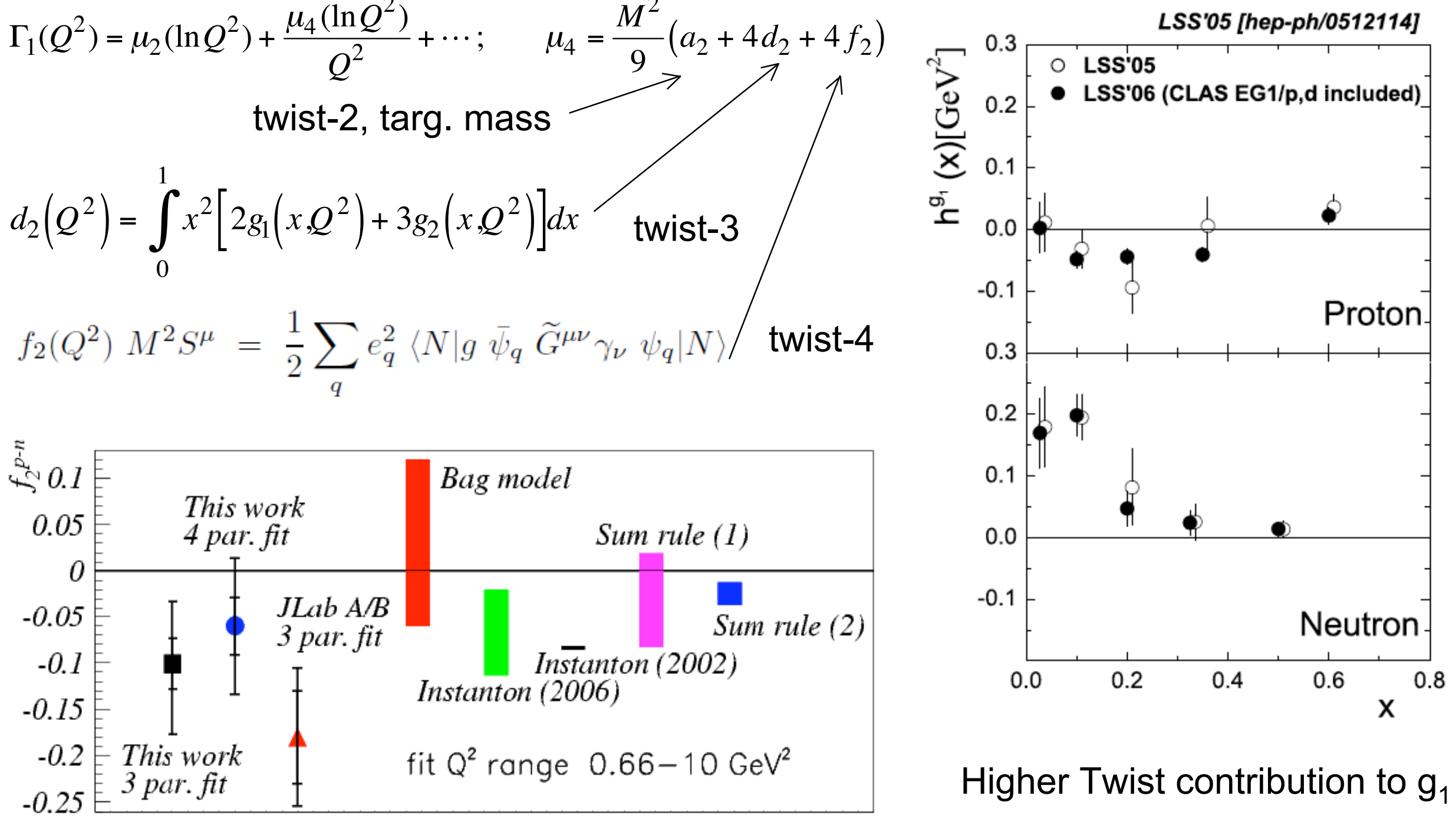

Higher Twist contribution to $g_{1}$ 


\section{Duality}

Long Distance Physics:

hadronic observables

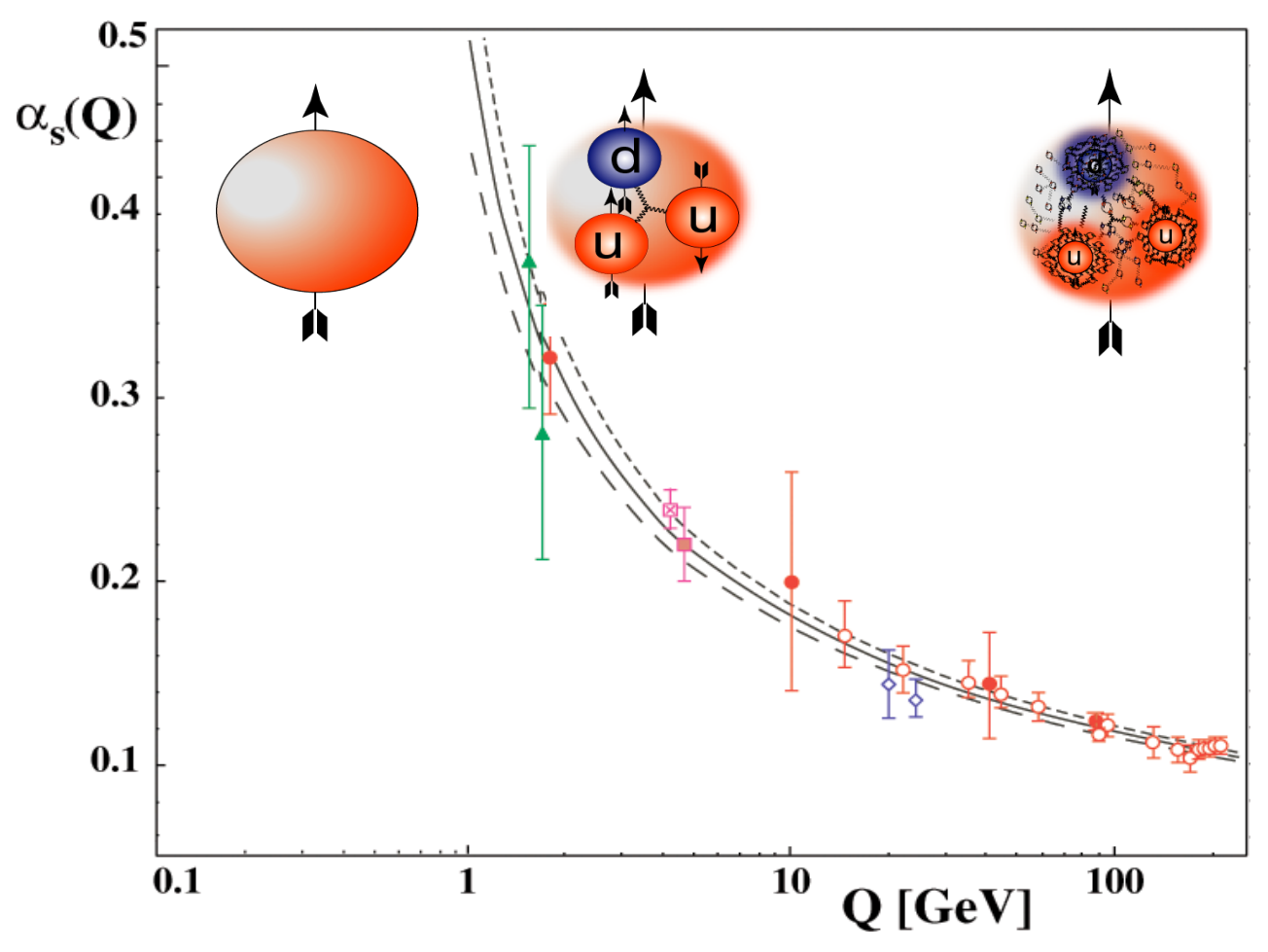

Famous Hall C Data

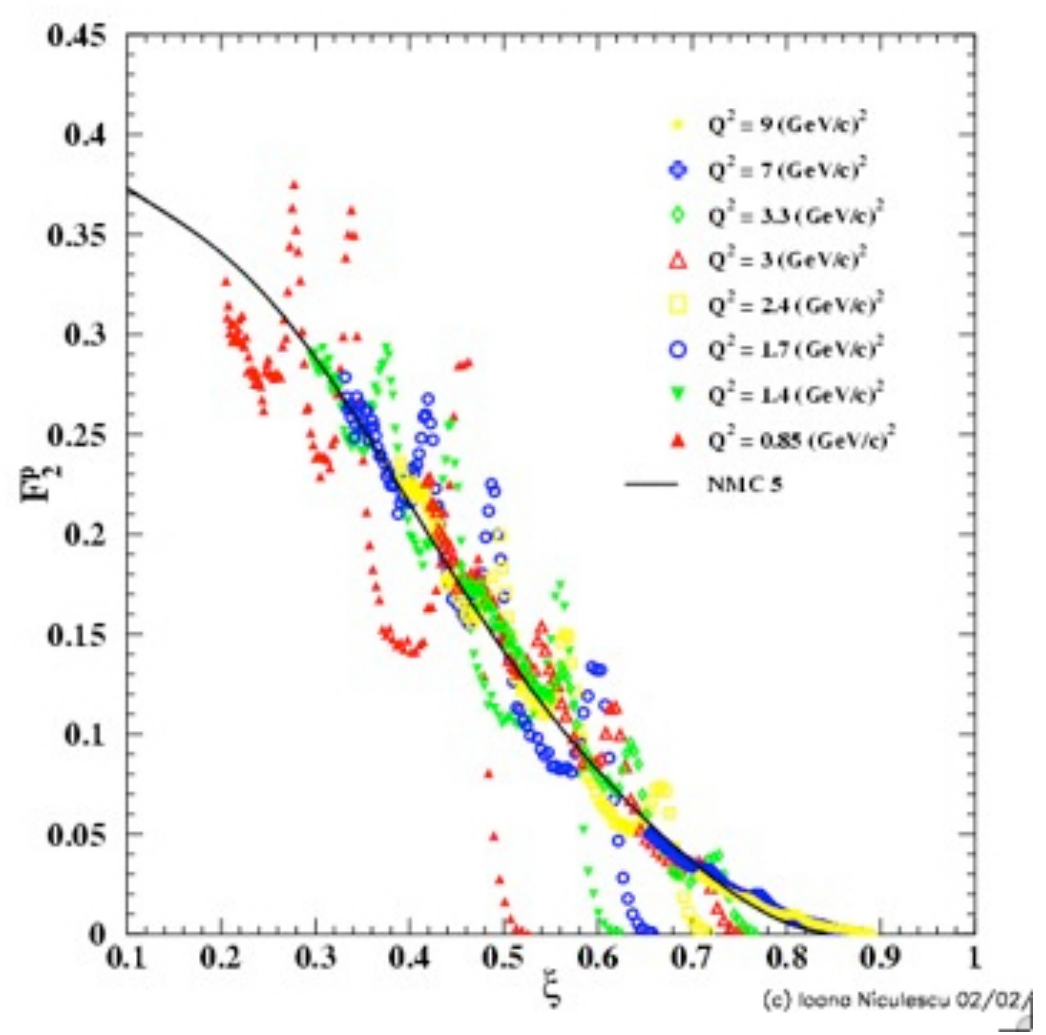

Asymptotically Free Quarks: regime of $\mathrm{pQCD}$ 


\section{Duality - New Results from Hall C}

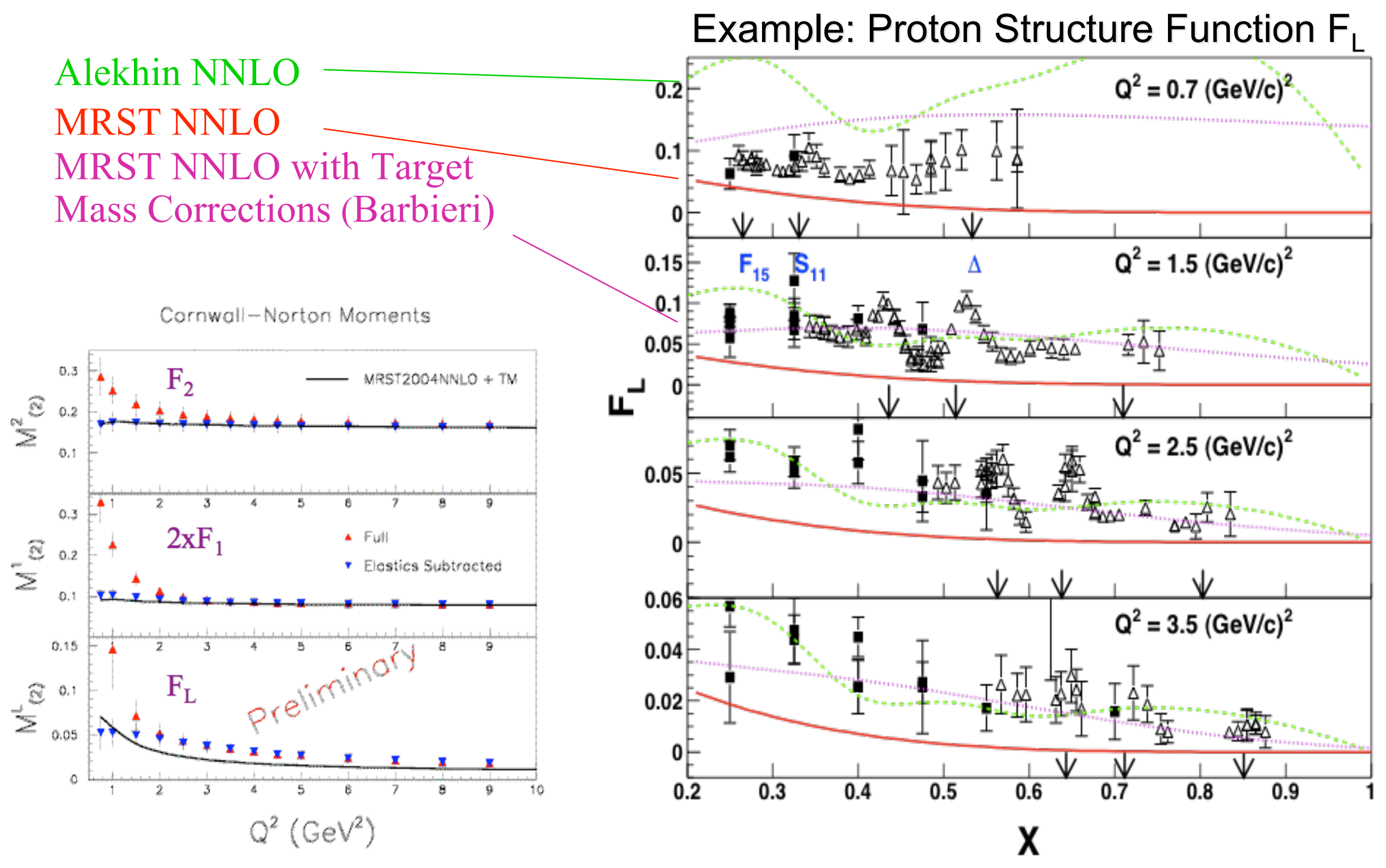




\section{Duality in SSFs}
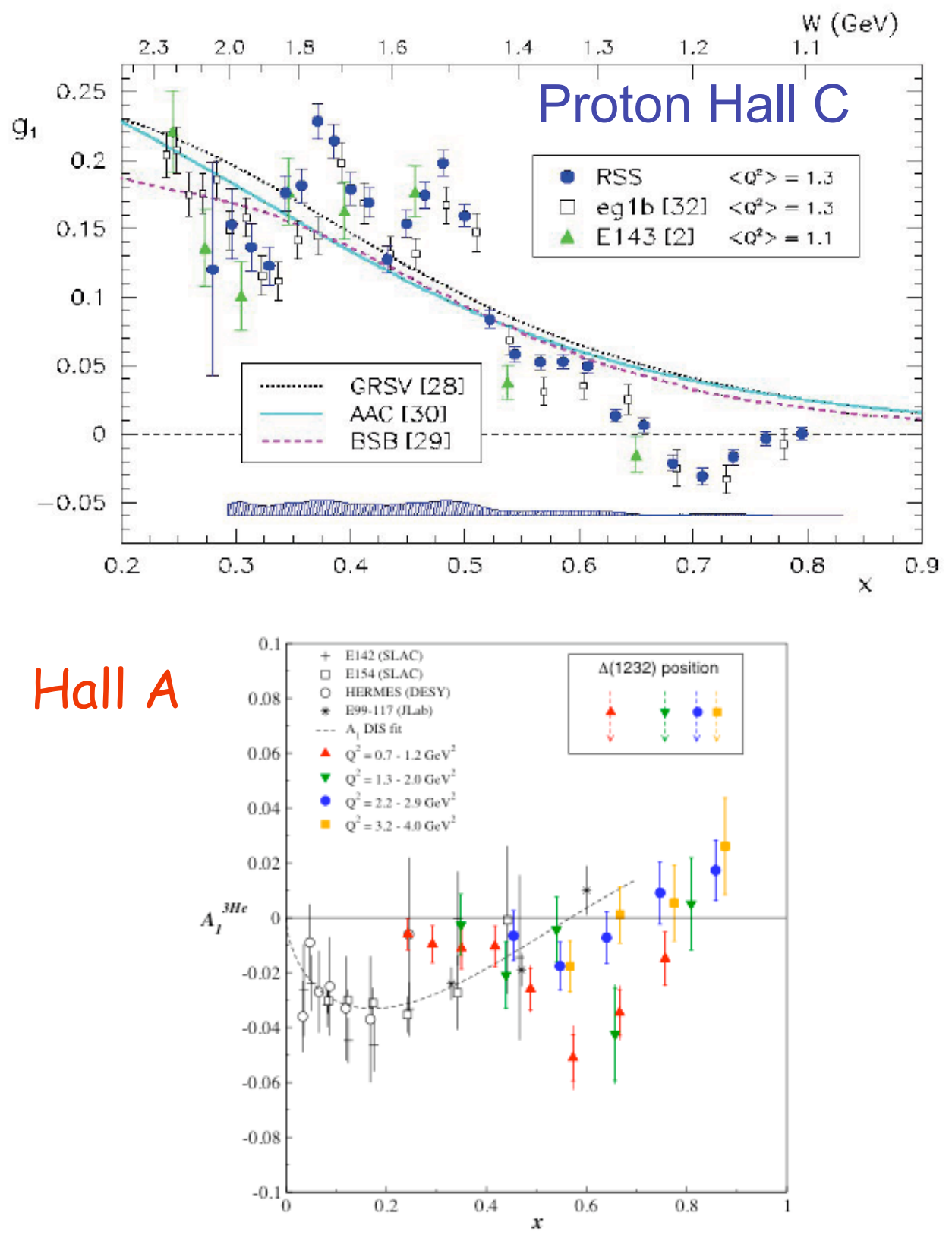

Hall $A$

P. Solvignon et al., PRL 101, 182502 (2008)

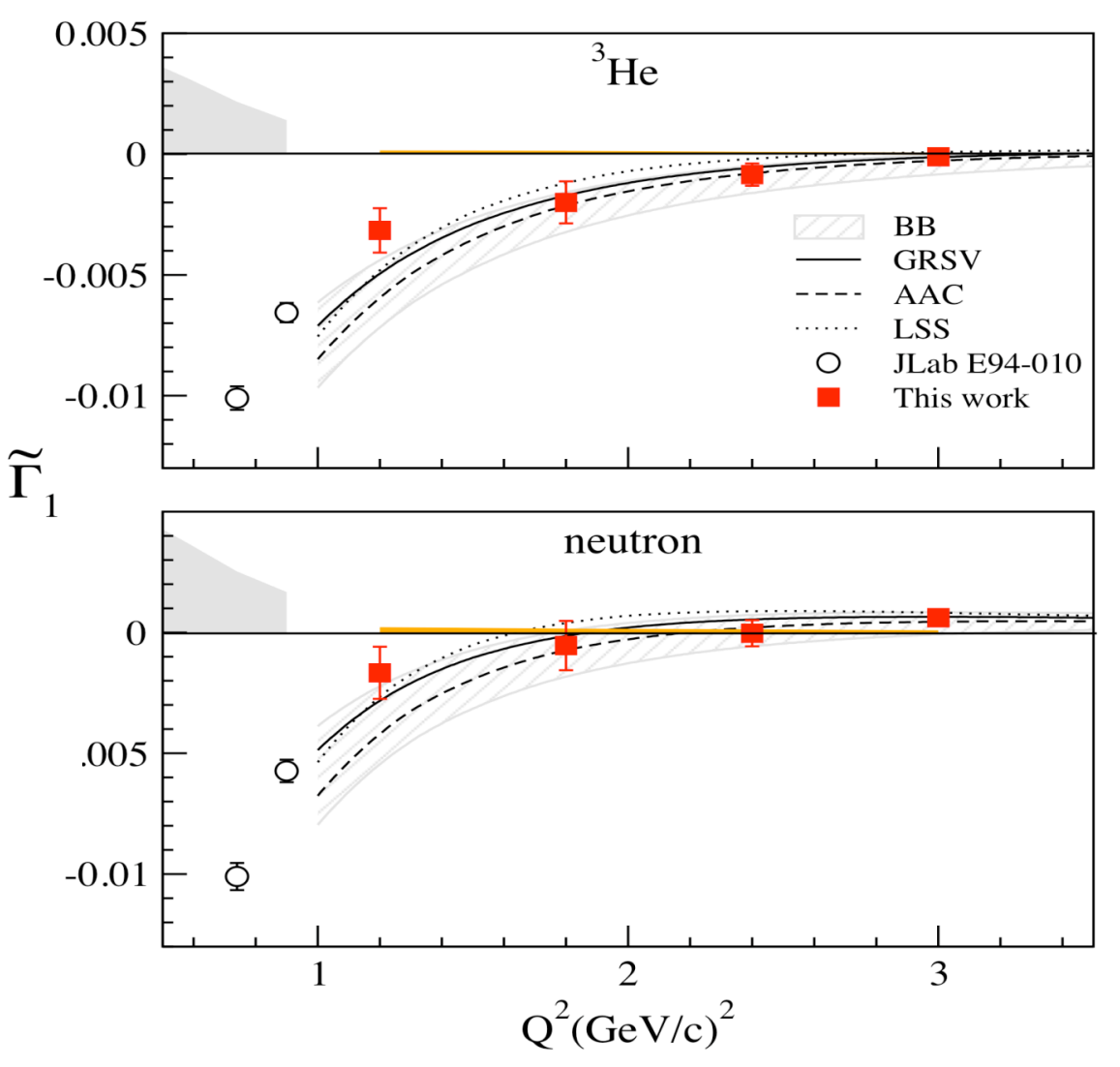

Target mass corrections were applied on PDFs 


\section{Duality in SSFs (CLAS)}

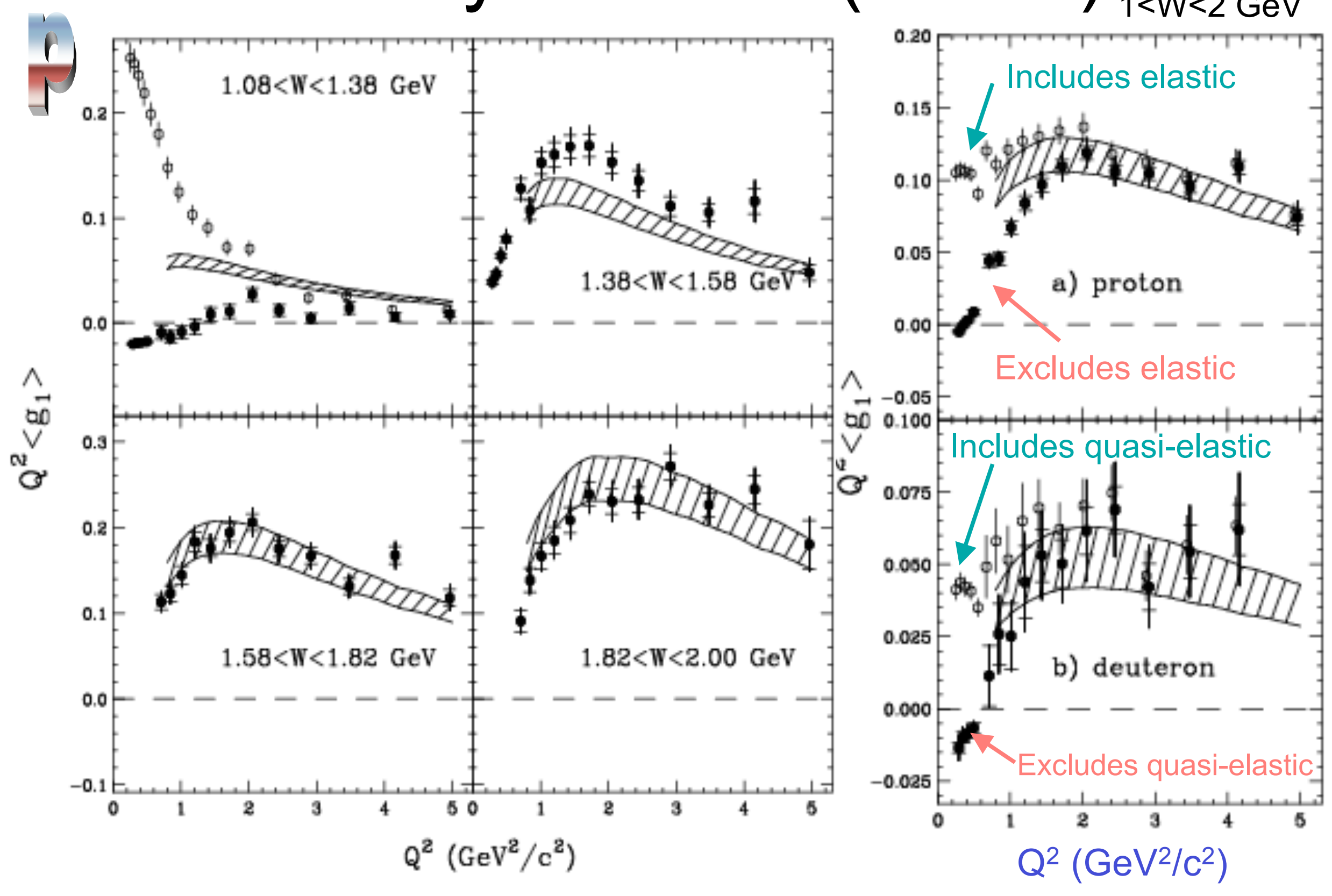




\section{New Data on the EMC Effect}

$\mathrm{A}\left(\mathrm{e}, \mathrm{e}^{\prime}\right)$ at 5.0 and $5.8 \mathrm{GeV}$ in Hall $\mathrm{C}$
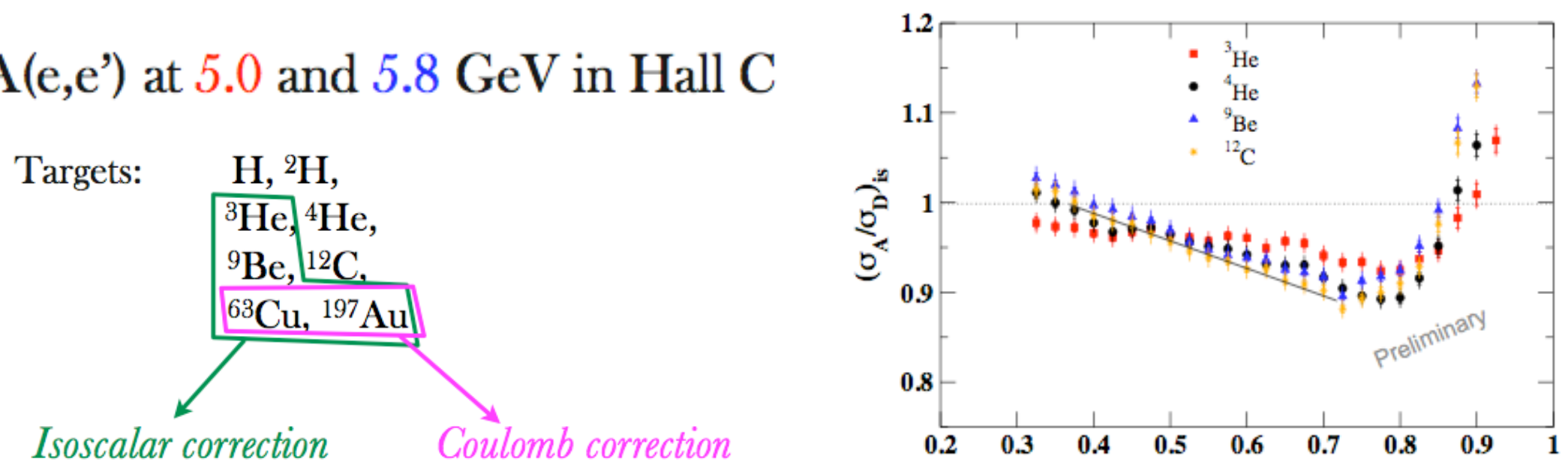

A or p-dependence?
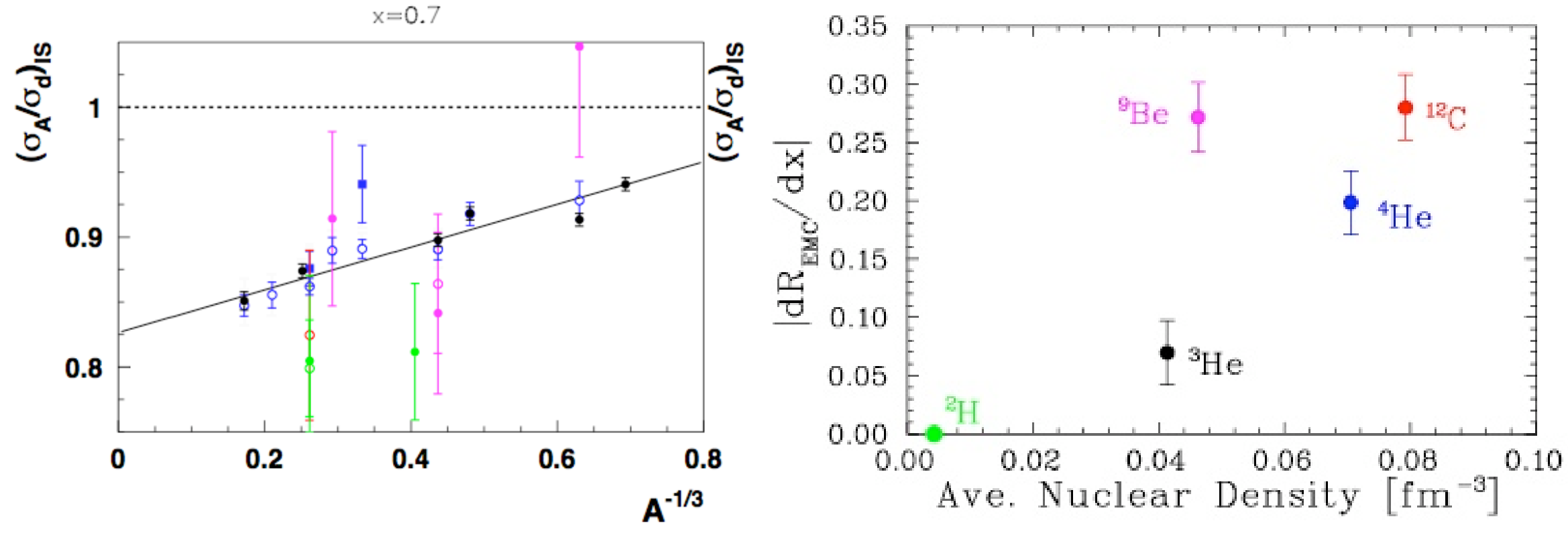


\section{SIDIS}

- By detecting the leading hadron in the final state together with the scattered electron, we can:

- tag the flavor of the struck quark

- select hard sub-processes through high $p_{\mathrm{T}}(12 \mathrm{GeV})$

- Measure $p_{\mathrm{T}}, \phi$ dependence of PDFs (higher twist)

- Learn about the 3-dimensional structure of the nucleon:

- Transversity

- Spin-momentum correlations (Sivers function, Boer-Mulders function, "pretzelosity")

- Learn about new fragmentation functions (Collins) 


\section{Unpolarized Results (CLAS):}

$H_{2}=\sum_{q} e_{q}^{2} q(x) \otimes D(z) \quad \mathrm{Q}^{2}=2.4 \mathrm{GeV}^{2}$

$<\cos \varphi>$ vs $p_{T}$
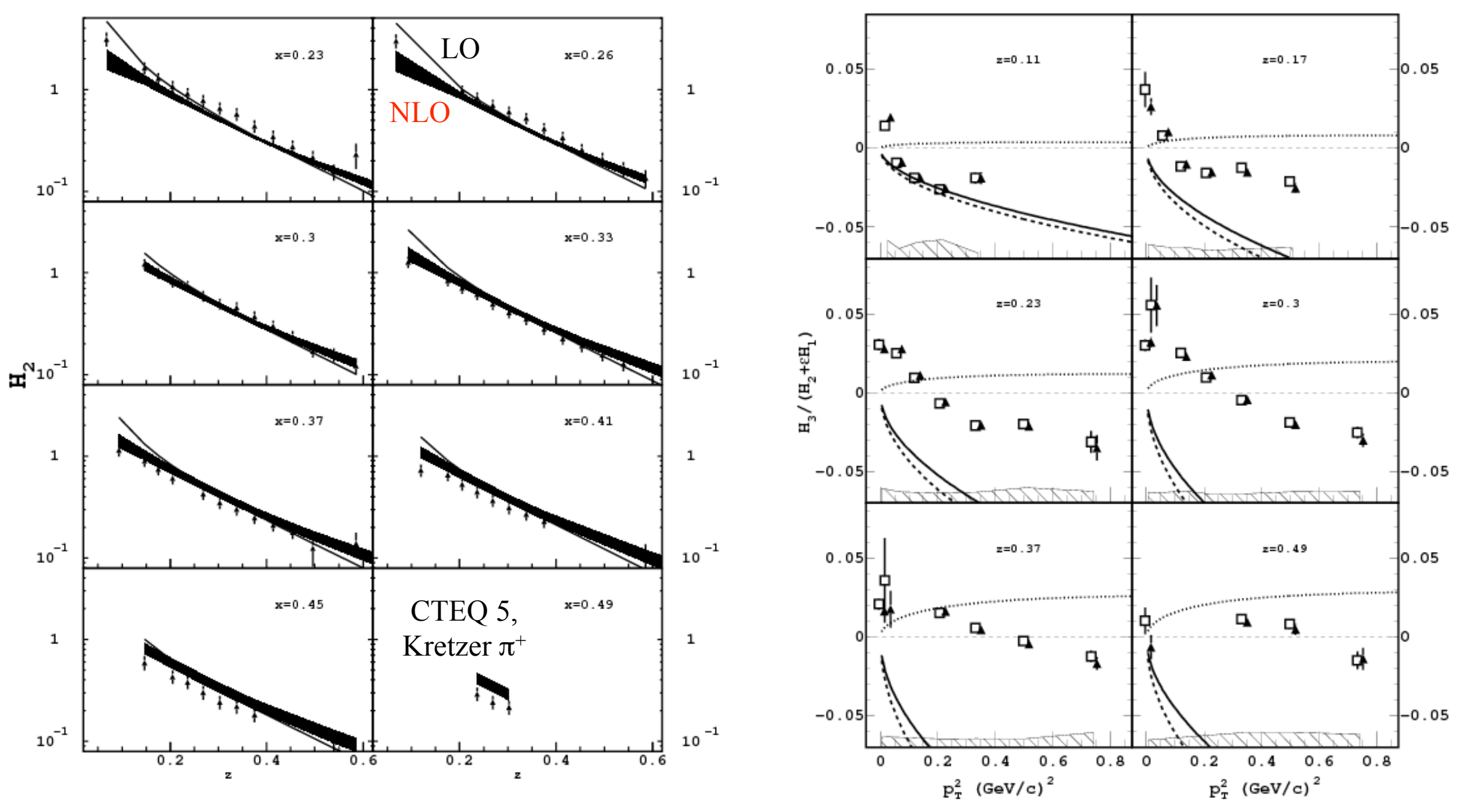


\section{Unpolarized Results (Hall C):}
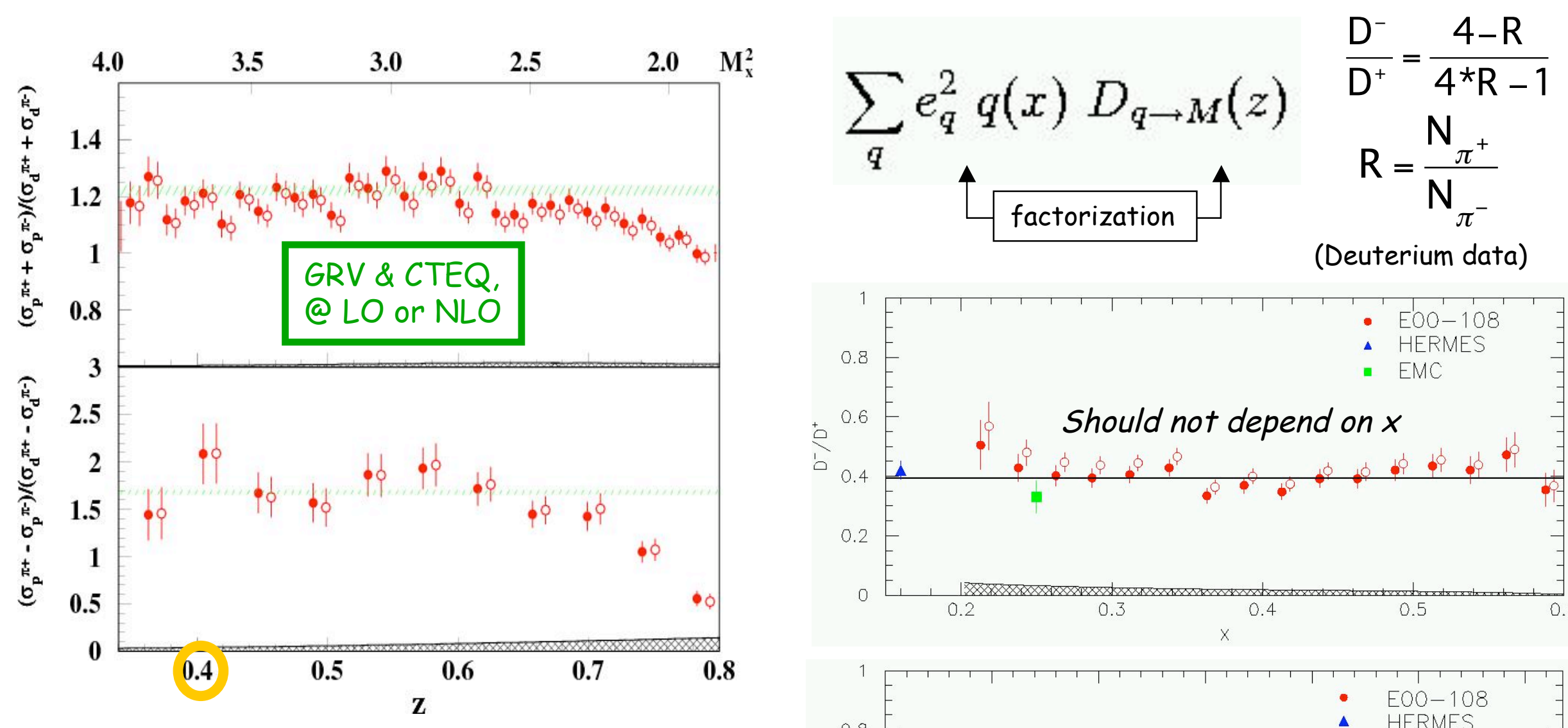

Closed (open) symbols reflect data after (before) events from coherent $\rho$ production are subtracted

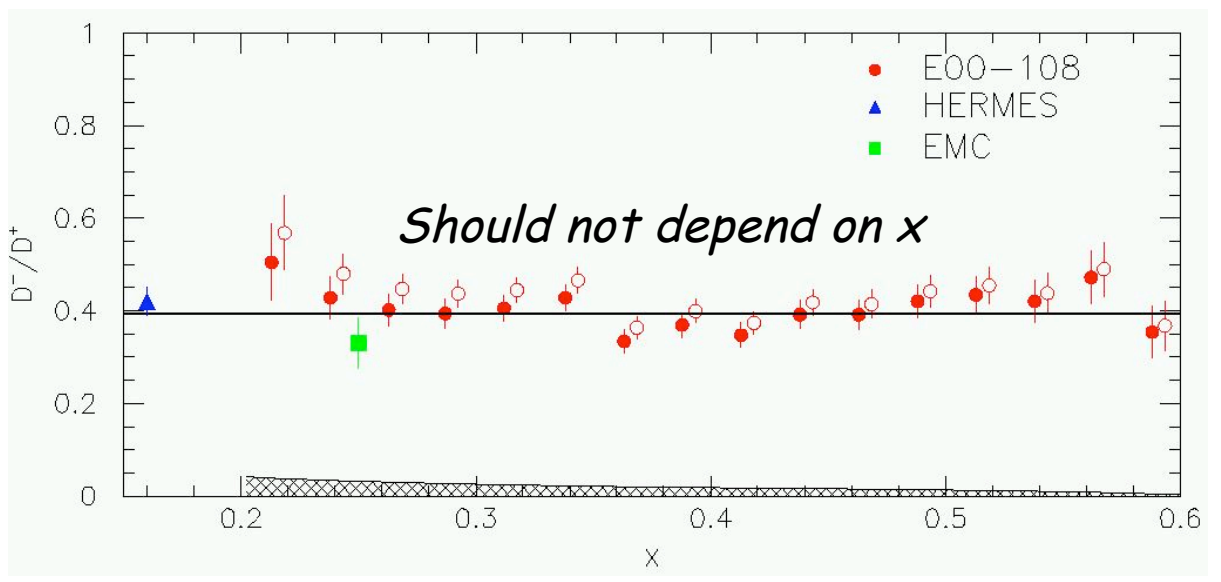

Good description for $p$ and $d$ targets for $0.4<z<0.65$

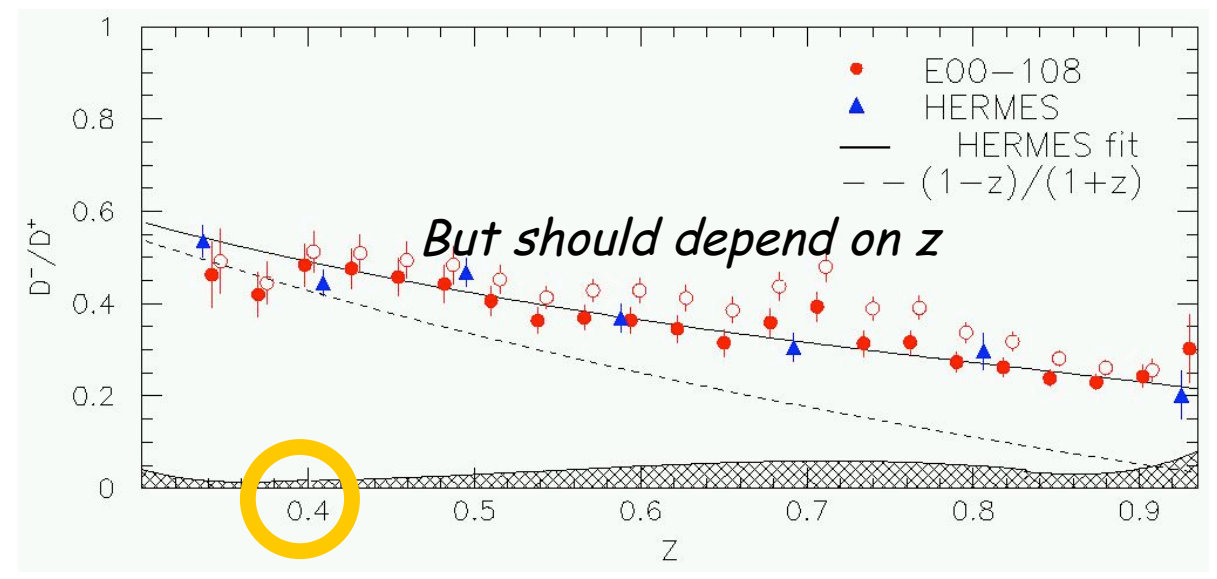




\section{Polarized Results - CLAS}
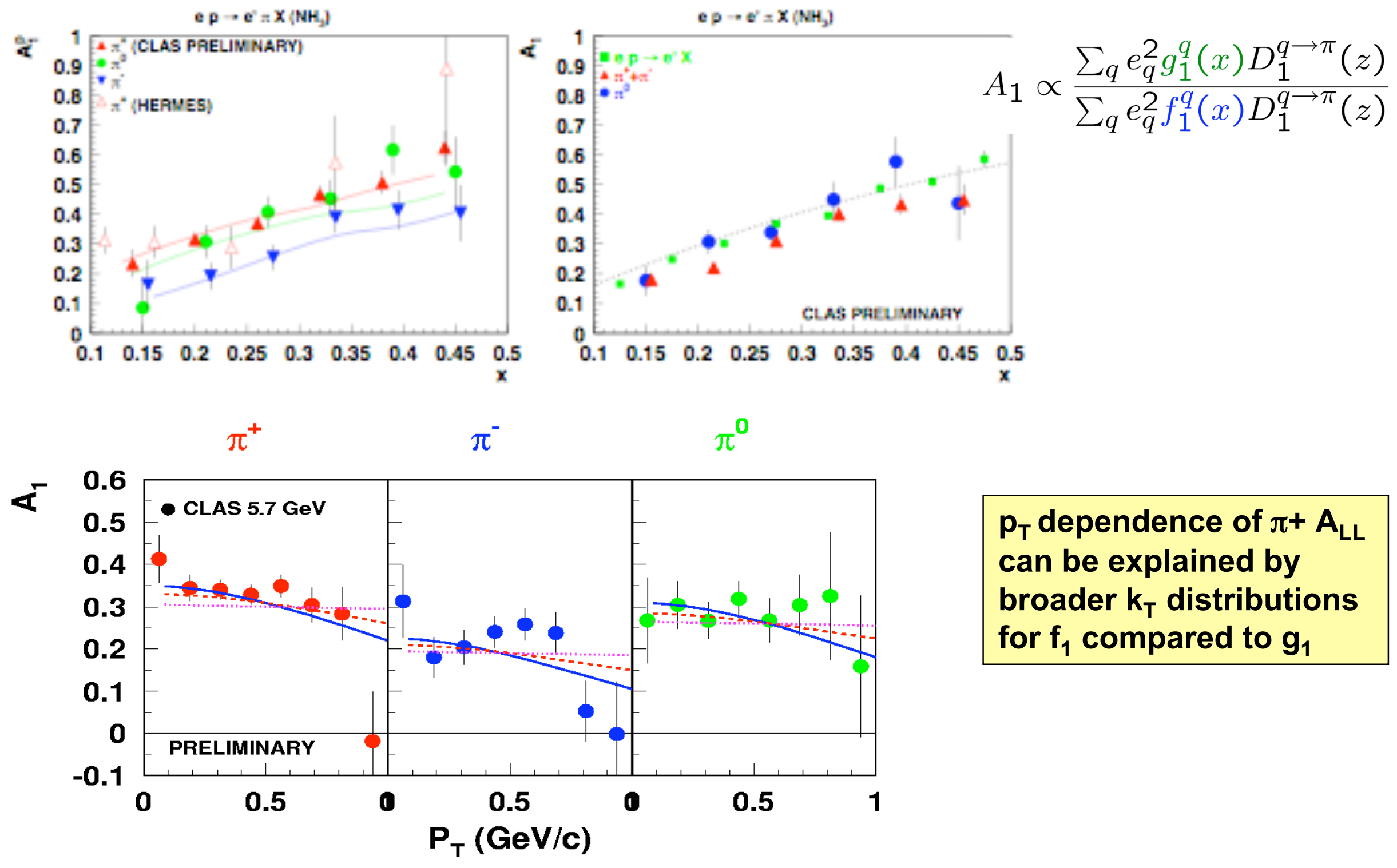

$p_{T}$ dependence of $\pi+A_{L L}$ can be explained by broader $\mathbf{k}_{\mathrm{T}}$ distributions for $f_{1}$ compared to $g_{1}$ 
E-05-113 with CLAS and longitudinal target Study semi-inclusive pion production, TMDs and $h_{1 L}^{\frac{1}{1}} H_{1}^{\perp}$ Collins fragmentation function as well as DVCS
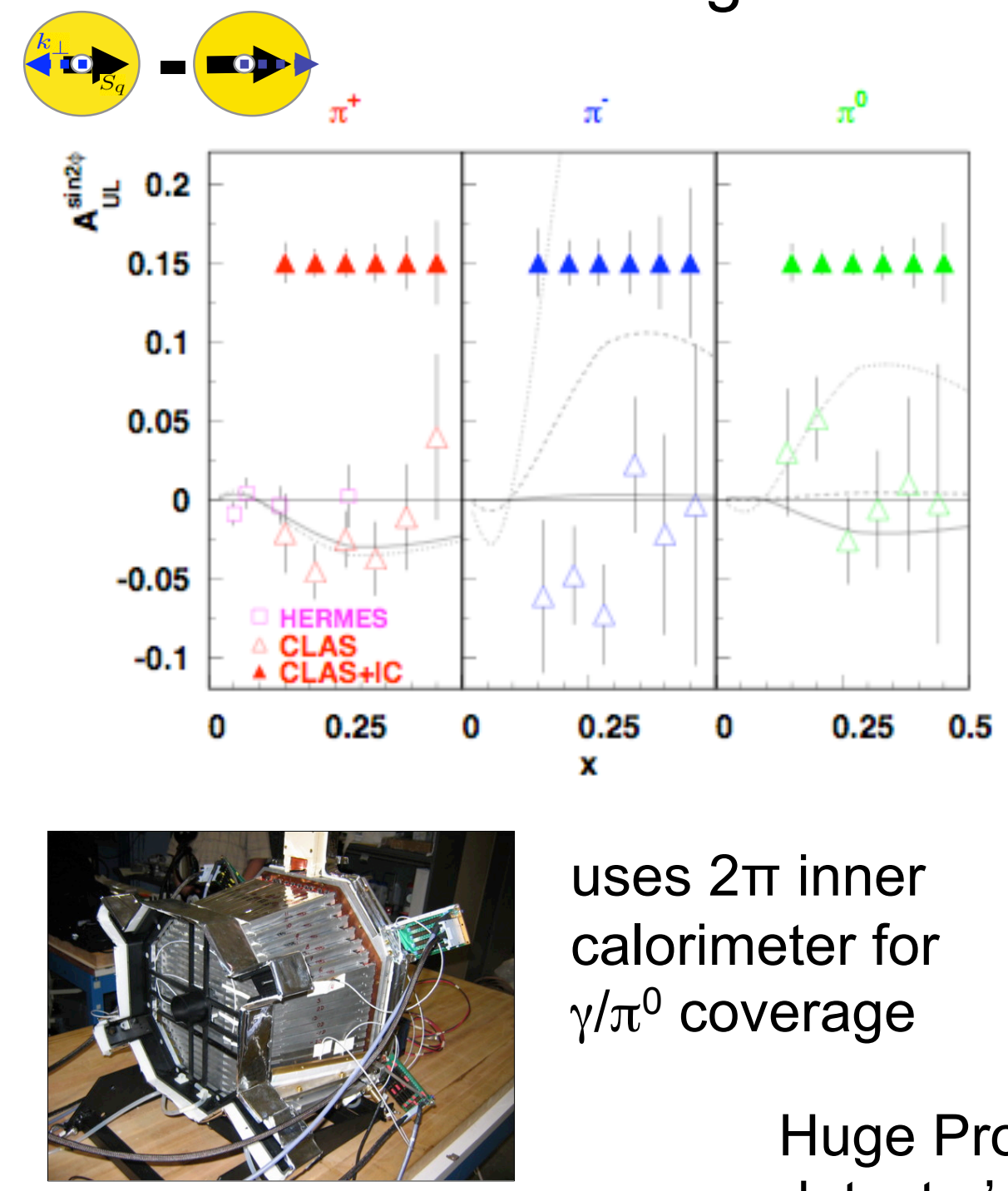

uses $2 \pi$ inner calorimeter for $\gamma / \pi^{0}$ coverage

Expected Precision for $\sin \phi$ and $\sin 2 \phi$ moments of target SSA 60 days $\left(P_{H}=75 \%\right)$

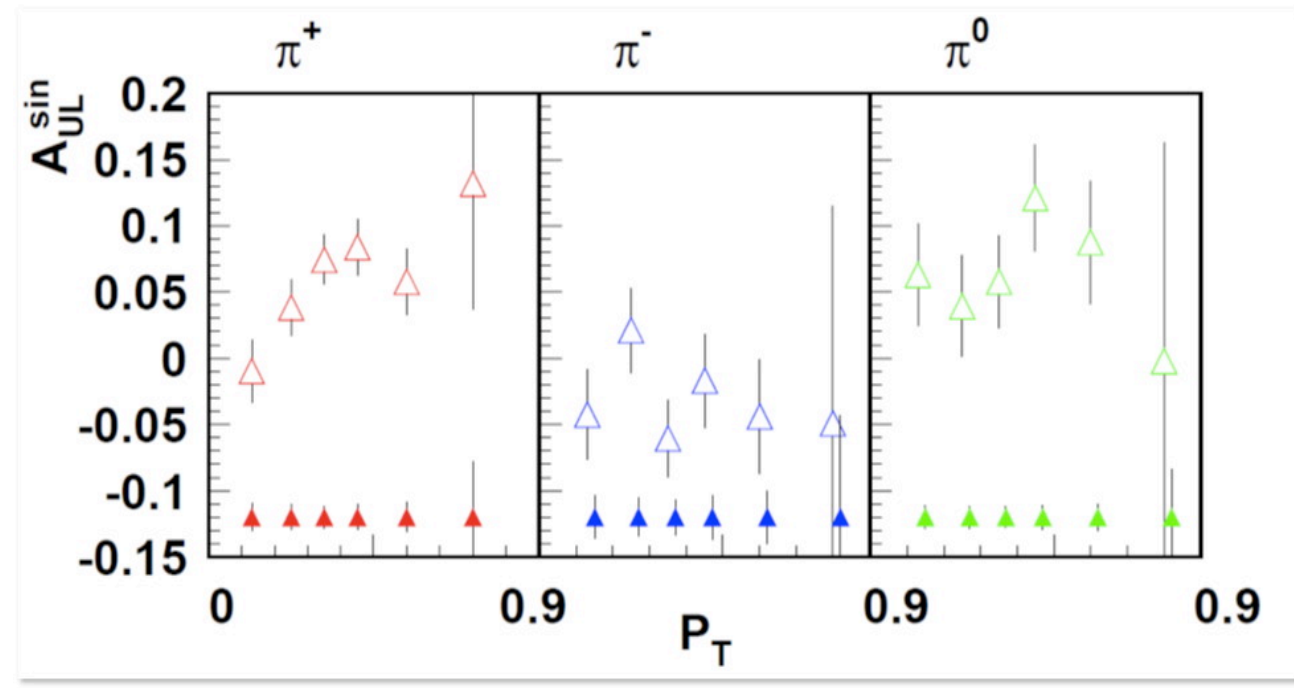

Huge Program for $12 \mathrm{GeV}: \mathrm{p}, \mathrm{d}$, ${ }^{3} \mathrm{He}$ targets, detect $\pi$ 's, K's, ...; transv. + long. SSAs, DSAs, ... 
E-08-015 with CLAS and transverse HD ice target Study Spin-Orbit correlations in Semi-Inclusive DIS and Sivers distribution function
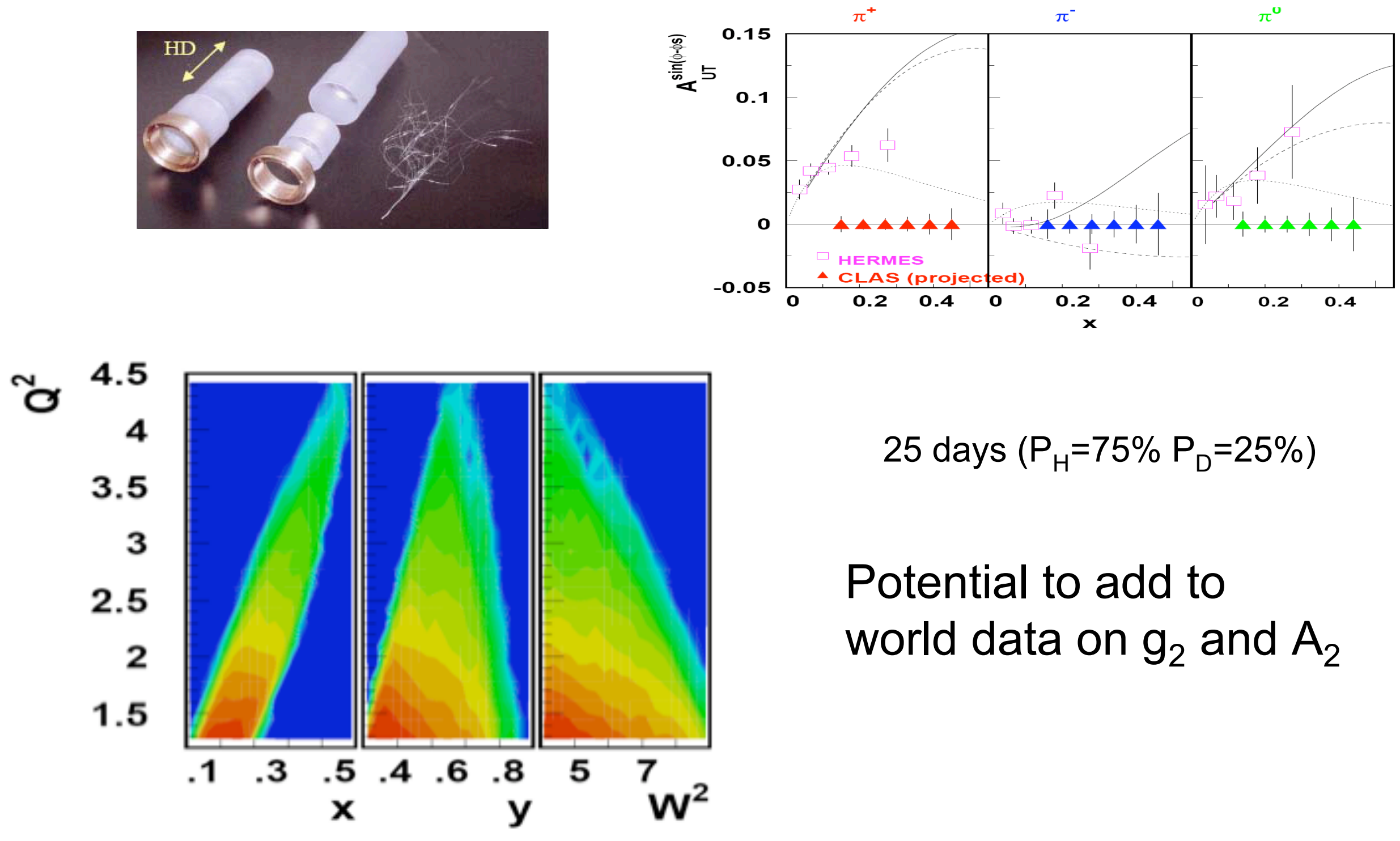

$$
25 \text { days }\left(P_{H}=75 \% P_{D}=25 \%\right)
$$

Potential to add to world data on $\mathrm{g}_{2}$ and $\mathrm{A}_{2}$ 
E06-010: Target Single-Spin Asymmetry in Semi-Inclusive $n^{\uparrow}\left(e, e^{9} \pi^{+/-}\right)$ Reaction on a Transversely Polarized ${ }^{3} \mathrm{He}$ Target
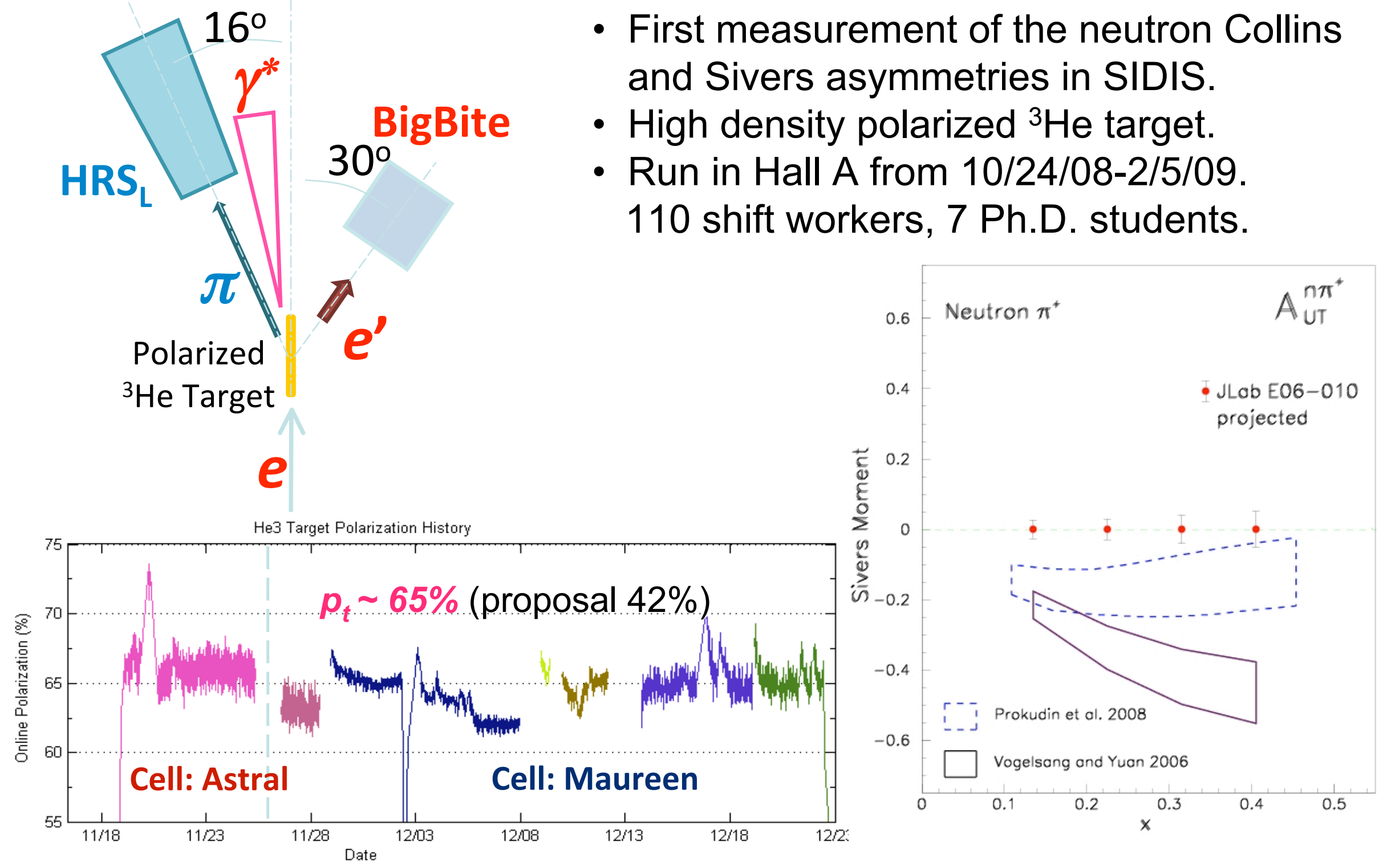


\section{Summary}

- There are huge number of interesting topics in (SI)DIS to pursue in "our little corner" of the kinematic plane

- Jefferson Lab has produced scores of high precision data on SFs and PDFs (TMD and integrated, especially at large $x$ ), moments, higher twist and duality, and nuclear effects

- Jefferson Lab at $12 \mathrm{GeV}$ (in 5 years) will complete the 3-dimensional quark picture of the nucleon for $x>0.1$ 